\title{
35. ANTARCTIC RADIOLARIA FROM THE SOUTHEAST PACIFIC BASIN, DEEP SEA DRILLING PROJECT, LEG 35
}

\author{
Fred M. Weaver, Antarctic Marine Geology Research Facility, \\ Department of Geology, Florida State University, Tallahassee, Florida
}

\section{INTRODUCTION}

During Leg 35 of the Deep Sea Drilling Project, a total of four sites were occupied (Figure 1). All sites drilled are located in the southeast Pacific sector of the Southern Ocean. Two sites, 322 and 323, are situated in the Bellingshausen abyssal plain, while the other two, Sites 324 and 325 , are located on the Antarctic continental rise. Fifty sediment cores were taken with a total recovered length of 193 meters.

Radiolarians occur at all sites drilled on DSDP Leg 35. Each site produced intermittent sequences of Neogene age sediments, and Hole 322 penetrated into Cretaceous material above its base. From the four sites, 237 samples were examined during this investigation; 137 were either totally barren of radiolarians or contained recrystallized assemblages. Radiolarians were commonly found in only 17 of the remaining samples which contained siliceous microfossils, and only 2 of these samples were pre-Pliocene in age. The general paucity and poor preservation of radiolarians examined from Leg 35 sediments is attributed to three factors: (1) high rates of sedimentation, (2) extreme water depths, and (3) significant diagenetic changes in prePliocene sediment sequences.

Due to lack of any continuous coring during Leg 35, no detailed biostratigraphic analysis and interpretation of regional changes in paleooceanography was possible, utilizing radiolarians.

\section{BIOSTRATIGRAPHIC FRAMEWORK}

The Antarctic radiolarian zonation proposed for the Plio-Pleistocene by Hays (1965) and Hays and Opdyke (1967) was modified and extended by Chen (1975) to include the entire Neogene. This zonation is utilized almost exclusively in this investigation.

In contrast to the zonal scheme of Hays (1965) and Hays and Opdyke (1967), the major advantages of Chen's zonation is that it employs fewer index species and uses only their latest or earliest occurrence rather than their last occurrence in significant numbers in defining zonal boundaries (Figure 2). The abundance of individuals of a species is related to a specific combination of oceanographic parameters. Therefore, the last "common" occurrence of a species may define a timetransgressive event, as the oceanographic province migrates in response to regional climatic changes. The diachroneity of radiolarian zones based upon the last frequent occurrence of index species was demonstrated by Weaver (1973) for Pliocene age sediments from the Antarctic.

\section{Pleistocene}

The only Pleistocene sedimentary sequence recovered during Leg 35 is at Site 324. The Antarctissa den- ticulata (= Omega Zone), and the Saturnalis circularis (= Chi Zone) zones of Chen (1975) are recognized in Core 1. The Stylatractus universus zone, which spans the early Brunhes magnetic epoch and precedes the Antarctissa denticulata zone could not be recognized in Core 1 . This may be the result of either a sedimentary hiatus in Core 1 or the sampling procedure. Stylatractus universus does occur in Core 1 , but only within the range of Saturnalis circularis.

It is especially significant to note that the $A$. denticulata and $S$. circularis zones proposed by Chen (1975) are useful in the Southern Ocean as far south as $69^{\circ} \mathrm{S}$ latitude.

\section{Pliocene}

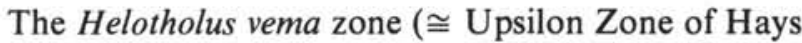
and Opdyke [1967]; see Figure 2) is identified in Pliocene sediments recovered at Sites 322, 323, and 325. The index species $H$. vema is rare and found sporadically at each site. Therefore, this zone is better recognized by the more consistent occurrence of Desmospyris spongiosa which has approximately the same stratigraphic range as $H$. vema in Southern Ocean sediments.

The Tau Zone of Hays and Opdyke (1967) is employed in Core 2 at Site 323. All samples examined from this interval lacked specimens of $H$. vema and $T$. b. spongothorax. However, they did contain numerous specimens of Triceraspyris coronatus $(=$ Triceraspyris $s p$.) whose upper range limit tentatively defines the top of the Tau Zone.

The Theocalyptra bicornis spongothorax Zone defined by Chen (1975) is recognized in one sample from Leg 35 sediments, 325-5, CC. However, in spite of the absence of specimens of $T . b$. spongothorax, the radiolarian assemblage that characterizes this zone also occurs in Samples 325-4, CC and 325-6, CC.

\section{Miocene}

The only well preserved Miocene radiolarian sequence recovered during Leg 35 was at Site 323. The middle Miocene Antarctissa conradae Zone of Chen (1975) could be recognized in Sample 325-3, CC. A number of samples from below Core 3, contain a typical middle Miocene radiolarian assemblage as illustrated by Chen (1975), but because of the absence of the index species, Calocyclas disparidens and Spongomelissa dilli, no other zones could be recognized in this middle Miocene sequence.

\section{RADIOLARIAN SITE SUMMARIES}

Tables 1-4 illustrate the occurrences of radiolarians identified from material recovered during Leg 35 . The following abbreviations are applied to species abundance and preservation: P (poorly preserved), M 


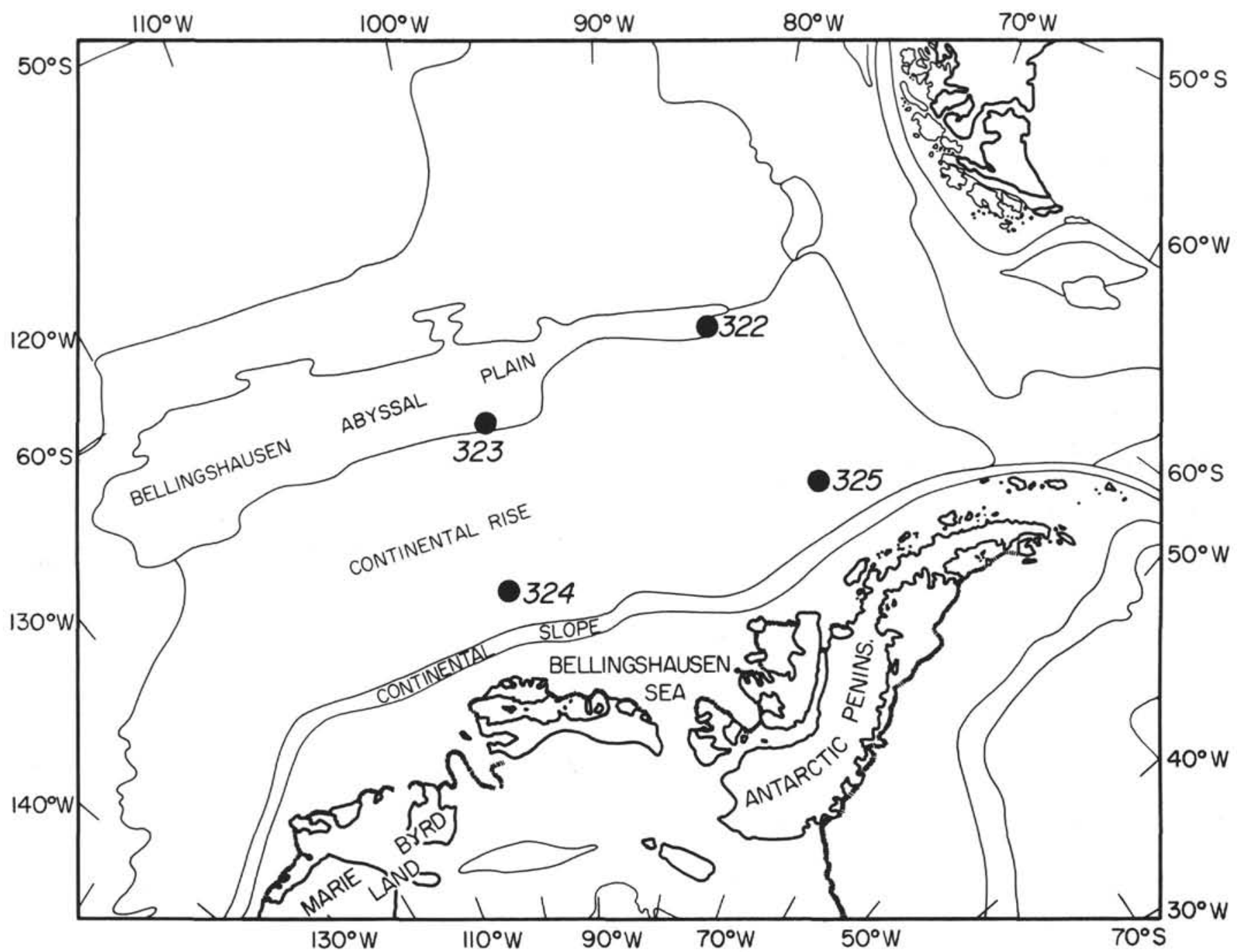

Figure 1. Location of drilling sites, Leg 35, Deep Sea Drilling Project, Southeast Pacific Basin.

(moderately well preserved), G (well preserved), A (abundant), C (common), F (few), R (rare), T (trace amounts-1 or 2 specimens per slide), and ? (questionable identification).

Site 322

$\left(60^{\circ} 01.45^{\prime} \mathrm{S}, 7^{\circ} 25.49^{\prime} \mathrm{W}\right.$; water depth $\left.5026 \mathrm{~m}\right)$

Site 322 is located at the eastern end of the Bellingshausen abyssal plain (Figure 1). Eleven "spot" cores with a total length of 34.4 meters were recovered before basement was encountered at a subbottom depth of 514 meters.

Poor to moderately well preserved radiolarians of latest Paleogene (?) and Neogene age occur sporadically throughout Site 322 sediments. Cores $1,2,4,6,8$, and 9 contain radiolarian assemblages with low abundances and species diversity. Other cores examined were either totally barren of radiolarians or contained specimens which were recrystallized.

Core 1, Section 1, 44-46 cm through Core 2, Section $2,97-99 \mathrm{~cm}$ contains moderately well preserved specimens of Desmospyris spongiosa, Helotholus vema, Eucyrtidium calvertense, Clathrocyclas bicornis, Prunopyle titan (?), and Lychnocanoma grande rugosum which are diagnostic of a Pliocene age for this interval (latest Gilbert-Gauss), and places it within the Upsilon Zone of Hays and Opdyke (1967) and the Helotholus vema Zone of Chen (1975) (Figure 2).

Core $322-3$ is totally barren of radiolarians, but the presence of poorly preserved specimens of Dendrospyris haysi, Eucyrtidium sp. aff. E. inflatum, Prunopyle hayesi, Stichocorys peregrina (?), Eucyrtidium calvertense, and Actinomma tanyacantha in Samples $322-2, \mathrm{CC}$ and 4, CC is diagnostic of a late Miocene age. No biostratigraphic zonal definition of this interval was possible due to the absence of any of the nominate index species proposed by Chen (1975).

The only radiolarians identified between Cores 5 through 10 occur in three samples: $322-8, \mathrm{CC}, 322-9-2$, $75-82 \mathrm{~cm}$, and $322-9$, CC (top). These are dated as Miocene on the basis of the occurrence of Dendrospyris haysi, Eucyrtidium calvertense, and E. cienkowskii group. Cores $322-5,7$, and 10 are totally barren, but Core 322-6 does contain some recrystallized specimens of the E. cienkowskii group.

\section{Site 323}

$\left(63^{\circ} 40.84^{\prime} \mathrm{S}, 9^{\circ} 59.69^{\prime} \mathrm{W}\right.$; water depth $\left.4993 \mathrm{~m}\right)$

Site 323 is located in the Bellingshausen abyssal plain just north of the Antarctic continental rise (Figure 1). 


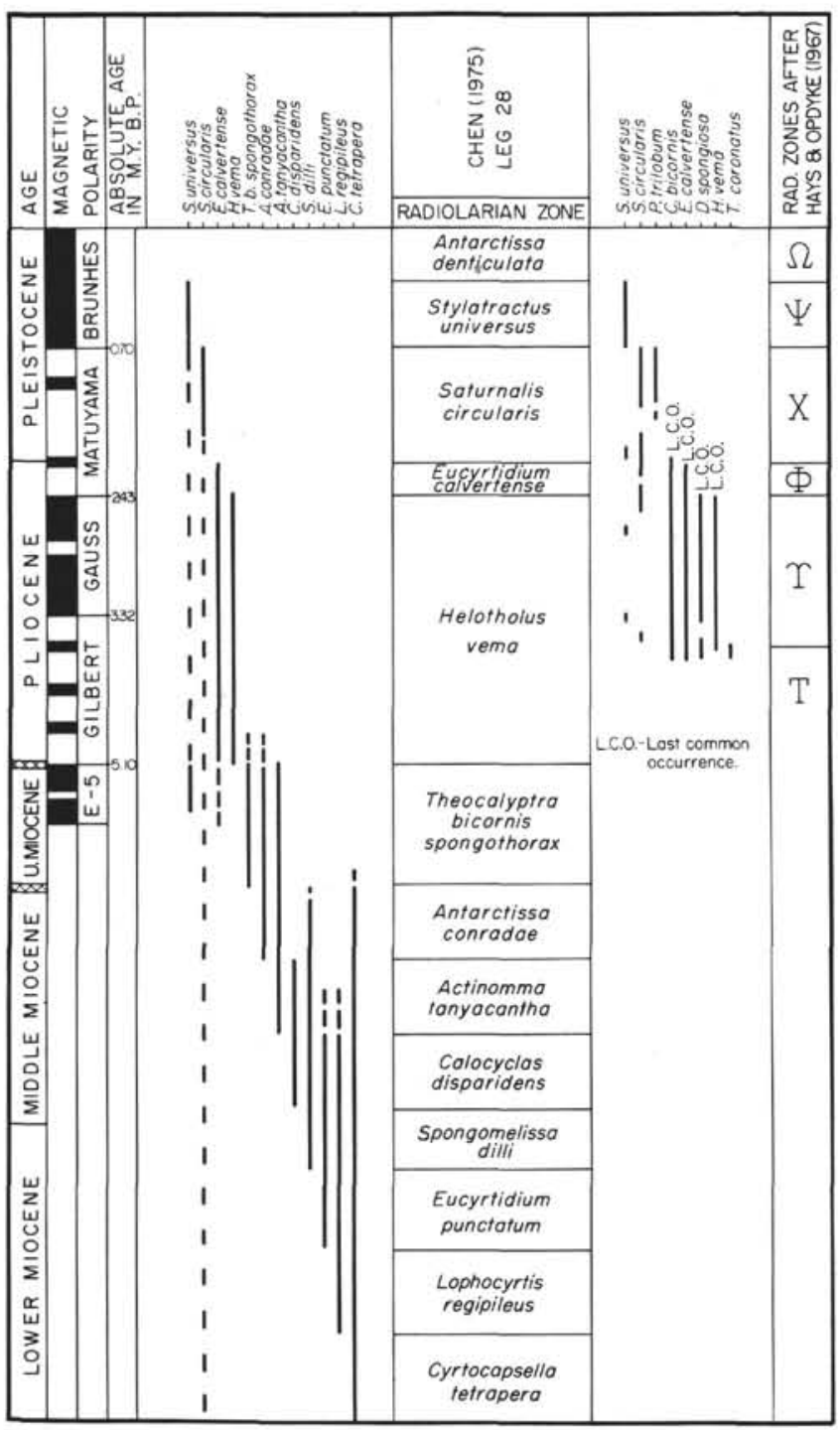

Figure 2. Biostratigraphic framework utilized during this investigation (from Chen, 1975, and Hays and Opdyke, 1967).

Eighteen sediment cores were taken at periodic intervals with a total recovered length of 76.4 meters. Basement was encountered at a subbottom depth of 701 meters.

Core 1, Section 1, 129-131 cm through Sample 2, CC contains a poor to moderately preserved Pliocene radiolarian assemblage. Although abundances of species fluctuate from common to rare, both the Helotholus vema Zone of Chen (1975) (Samples 323-1-2, 107$109 \mathrm{~cm}$ through 323-1, CC, and the Tau Zone of Hays and Opdyke (1967) (Samples 323-2-1, 65-67 cm through 323-2, CC) are recognized (Table 2).

Core 3 , Section 1 and Core 3 , Section 2 contain a few, moderately well preserved radiolarians which are long ranging and diagnostic of a Miocene age. Samples 323$3-2,130-132 \mathrm{~cm}$ and $323-3, \mathrm{CC}$ also contain numerous specimens of Orosphaerid (spines only) and Collosphaerid radiolarians.

A typical middle Miocene radiolarian assemblage which is well preserved occurs in Sample 3, CC. Com- mon specimens representing the lower portion of the Antarctissa conradae Zone of Chen (1975) are present. Distinctive species represented are (in order of decreasing abundance) Cyrtocapsella tetrapera (30\%-40\% of the entire fauna), Lychnocanoma sphaerothorax, Antarctissa conradae, Prunopyle hayesi, Eucyrtidium cienkowskii group, Actinomma tanyacantha, Dendrospyris megalocephalis, Cyrtocapsella cornuta (?), C. isopera (rare), Amphistylus angelinus, and Sethoconus sp. (Table 2).

Sample 4, CC through Sample 6-1, 118-121 cm are essentially barren except for Orosphaerids and a few of the more resistant high-latitude Miocene radiolarians which include $C$. tetrapera, $P$. hayesi, L. sphaerothorax, and $A$. angelinus. None of the previously established radiolarian zones could be recognized within this interval.

The material recovered between Samples 323-6, CC and $323-8-1,98-100 \mathrm{~cm}$ is middle Miocene in age. Preservation and abundance of specimens vary depending on the sediment lithologies. Based upon the cooccurrence and relatively high abundances of Lophocyrtis golli, Lophocyrtis regipileus, and Eucyrtidium punctatum group, the presence of Cyrtocapsella isopera, and the absence of Actinomma tanyacantha, this inter$\mathrm{val}$ is assigned to the upper portion of the Spongomelissa dilli through the Calocyclas disparidens zones of Chen (1975) or early middle Miocene. Other species occurring within this interval include, Prunopyle hayesi, Collosphaerids, Orosphaerids, Eucyrtidium cienkowskii group, Amphistylus angelinus, Dendrospyris haysi, Sethoconus sp., Cyrtocapsella tetrapera, and Stylacontarium bispiculum.

Middle Miocene index species, Spongomelissa dilli, Calocyclas disparidens, and Thyrsocyrtis clausa, were not recognized in any of the 11 samples analyzed from Cores 6 through 8, suggesting that the middle Miocene zonal scheme proposed by Chen (1975) may need some revision or redefinition in order for it to be applicable throughout the entire Southern Ocean region.

Thirty-eight samples examined between Samples 323$8, \mathrm{CC}$ and $323-18-2,138-140 \mathrm{~cm}$ are entirely barren of radiolarians.

Commonly occurring but poorly preserved Cretaceous radiolarians were found in Sample 323-18-3, 63-65 cm through Section 323-18-6. The siliceous tests are completely recrystallized, and only limited morphological characteristics are preserved (test outline, number of segments, etc. A high percentage of the specimens examined belong to the genus Dictyomitra Zittel (1876), but species definition is impossible to establish due to the poor state of preservation.

\section{Site 324}

$\left(69^{\circ} 03.21^{\prime} \mathrm{S}, 98^{\circ} 47.20^{\prime} \mathrm{W}\right.$; water depth, $\left.4449 \mathrm{~m}\right)$

Site 324 is located on the upper continental rise of Antarctica (Figure 1). Ten cores, with a total recovery of 48 meters, were taken before the site was abandoned at a subbottom depth of 199 meters.

Sixty-five samples were examined for radiolarians from the first nine cores. Forty-eight of these samples from between 324-1, CC and 324-9-1 are totally barren of any radiolarian remains. 
TABLE 1

Radiolarians at Site 322

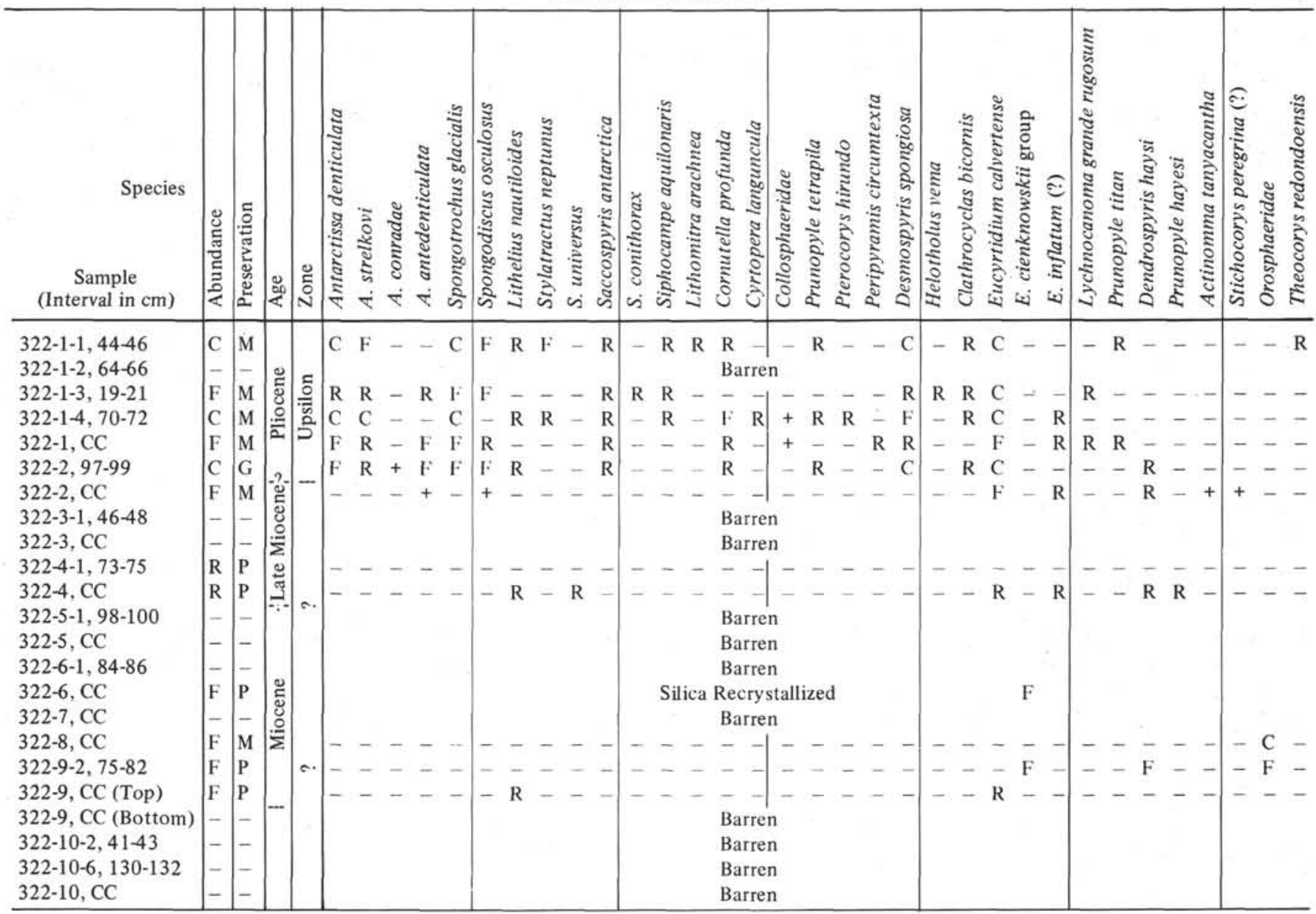

Only the core catchers of Core 9, and Core 1 contain any radiolarians.

Core 324, Section 1 is of Pleistocene age, and two biostratigraphic zones defined by Chen (1975) are recognized. Samples 324-1-1, 143-145 cm through 324$1-2,53-55 \mathrm{~cm}$ fall within the Antarctissa denticulata Zone, while the Saturnalis circularis Zone extends from Samples 324-1-2, 138-140 cm through 324-1-6, 128-130 $\mathrm{cm}$. Most samples from Core 1 contain the typical Quaternary Southern Ocean radiolarian assemblage which consists of Antarctissa denticulata, A. strelkovi, Stylatractus neptunus, Lithelius nautiloides, Spongotrochus glacialis, Spongodiscus osculosus, Saccospyris antarctica, Triceraspyris antarctica, Stylodictya validispina, Spongoplegma antarcticum, Peripyramis circumtexta, Prunopyle antarctica, Cycladophora davisiana, and Spongurus pylomaticus.

The Stylatractus universus Zone of Chen (1975) is not recognized in Core 324-1, although this species was found in the Saturnalis circularis Zone.

Sample 324-9, CC contains rare and moderately well preserved radiolarians diagnostic of an early Pliocene age (Table 3), but this age determination is quite uncertain because the hole was collapsing while Core 9 was being taken. Even with the barren nature of the sediments at Site 324, down-hole contamination from radiolarian-rich stringers between the coring gaps cannot be ruled out.

\section{Site 325}

$\left(65^{\circ} 02.79^{\prime} \mathrm{S}, 7^{\circ} 40.40^{\prime} \mathrm{W}\right.$; water depth $\left.3745 \mathrm{~m}\right)$

Site 325 lies on the upper continental rise northwest of the central Antarctic peninsula. Ten "spot" cores were taken throughout the 718 meters of sediment penetrated, and 34.4 meters were recovered (Figure 1).

Radiolarians were found in all cored intervals at Site 325. Preservation is poor to moderate while species abundances and diversity are generally low. Cores 7, 8, 9 , and 10 contain abundant radiolarians, but diagenetic effects within the claystones have resulted in partial or complete test recrystallization. Identification and age determinations within these intervals were therefore difficult and may be unreliable.

Core 1, Section 1, 18-20 cm through Core 3, CC contains a typical Pliocene radiolarian assemblage and can be assigned to the Helotholus vema Zone of Chen (1975) (Table 4). Species include Antarctissa denticulata, $A$. strelkovi, Spongotrochus glacialis, Spongodiscus osculosus, Triceraspyris antarctica, Saccospyris antarctica, Prunopyle tetrapila, P. titan (?), Lithelius nautilioides, Cornutella profunda, Desmospyris spongiosa, Eucyrtidium calvertense, and Helotholus vema. 
Although the index species $H$. vema occurs only sporadically throughout this interval, $D$. spongiosa, which has approximately the same stratigraphic range as $H$. vema, does occur consistently through the entire interval, thus restricting it to the Pliocene.

Samples 325-4, CC, 325-5-1, 102-104 cm, 325-5, CC, and $325-6, C C$ are assigned to the Theocalyptra bicornis spongothorax Zone of Chen (1975). The top of this zone is placed between $325-3, \mathrm{CC}$ and $325-4, \mathrm{CC}$ at the first occurrence of Helotholus vema. Although Theocalyptra bicornis spongothorax only occurs in Sample 5, CC, the co-occurrence of the Pliocene form of Stylatractus universus, Prunopyle hayesi, Stichocorys peregrina (?), Antarctissa conradae, and A. antedenticulata (see Table 4), throughout the other samples examined within this interval, restricts them to the $T . b$. spongothorax Zone of Chen.

Radiolarian age determinations from material recovered from Cores 7 through 10 are difficult due to recrystallization of the specimens present. Two species could positively be identified within this interval, Eucyrtidium cienkowskii group and Prunopyle hayesi (Table 4). Cores 9 and 10 contain recrystallized specimens of what appears to be Cannartus mammiferus, based on their rough morphological appearance. Most details are obscured, but what may be remanent protuberances can be seen on the sides of the cortical shells of many specimens (Plate 5, Figure 8). Sample 10$1,90-92 \mathrm{~cm}$ contains several specimens closely resembling Cannartus prismaticus (Plate 5, Figure 9; note the outline of the polar columns and cortical shell).

If these identifications are correct, Cores 9 and 10 could possibly range in age from upper Oligocene through the middle Miocene, based upon the defined ranges of Cannartus mammiferus and $C$. prismaticus in lower latitudes.

\section{SYSTEMATIC DESCRIPTIONS}

The classification proposed by Riedel (1967a, b; 1971) is followed wherever possible. Synonomies of taxa identified are not always complete and are often restricted to the original description and revised concept of the species.

Since the reports on Antarctic radiolarians from DSDP Legs 28 and 29 were completed independently, two completely different biostratigraphic zonations and illustrations of new taxa have emerged (Petrushevskaya, 1975; Chen, 1975). Numerous conflicting generic and species designations for pre-Pliocene Tertiary radiolarians from the Southern Ocean have added to the confusion created by inflated synonomies. In order to clarify these data for researchers dealing with Antarctic radiolarian taxa, Table 5 is included prior to the systematic descriptions in this report. This illustrates some important radiolarian taxa whose descriptions apparently have been duplicated.

Although the Leg 29 initial reports were published prior to Leg 28, the new taxonomy and biostratigraphy utilized in this report follow that presented by Chen in his Leg 28 studies. A preprint from the Leg 28 report was available at the time this investigation was being completed.

Plates 6-9 contain scanning electron micrographs taken on an International Scientific Instruments Super-Mini Scan. Wherever possible, both light micrographs and scanning electron micrographs of the same species, or species group, are illustrated in order to provide maximum morphological detail.

Subclass RADIOLARIA Müller, 1858

Order POLYCYSTINA Ehrenberg, 1838, emend. Riedel, 1967b

Suborder SPUMELLARIA Ehrenberg, 1875

Familty ACTINOMMIDAE Haeckel

Actinommidae Haeckel, 1862, emend. Riedel, 1967b
Genus ACANTHOSPHAERA Ehrenberg

Acanthosphaera Ehrenberg, 1858, p. 12.

Acanthosphaera sp.

Acanthosphaera sp. Hays, 1965, p. 169, pl. II, fig. 8; Chen, 1975, p. 509 , pl. 22 , fig. 5 .

Abundance: Rare to few.

Occurrence: Pleistocene, Saturnalis circularis Zone (Site 324).

\section{Genus ACTINOMMA Haeckel}

Actinomma Haeckel, 1862, p. 440.

\section{Actinomma tanyacantha Chen}

Actinomma tanyacantha Chen, 1975, p. 487, pl. 11, fig. 5, 6. Abundance: Rare to few.

Occurrence: Earliest Pliocene and middle Miocene (Site 323).

\section{Actinomma yosii Nakaseko}

(Plate 1, Figure 1)

Actinomma yosii Nakaseko, 1959, p. 10, pl. II, fig. 8a, b. Abundance: Rare to few.

Occurrence: Pleistocene.

\section{Genus AMPHISTYLUS Haeckel}

Amphistylus Haeckel, 1881, p. 452.

Amphistylus angelinus (Campbell and Clark)

Stylosphaera angelina Campbell and Clark, 1944, p. 12, pl. 1, fig. 1420.

Axoprunum angelinum (Campbell and Clark) Kling, 1973, p. 634, pl. 6, fig. 18.

Amphistylus angelinus (Campbell and Clark) Chen, 1975, p. 503, pl. 21, fig. 3, 4 .

Abundance: Rare to few.

Occurrence: Middle Miocene (Site 323).

\section{Genus DRUPPATRACTUS Haeckel}

Druppatractus Haeckel, 1887, p. 324.

\section{Druppatractus sp.}

(Plate 5, Figures 1, 2; Plate 6, Figure 1)

Druppatractus sp. Chen, 1975, p. 505, pl. 20, fig. 11, 12 .

Remarks: A few specimens of this species were observed in early Pliocene sediments at Site 323.

\section{Genus ECHINOMMA Haeckel}

Echinomma Haeckel, 1881, p. 453.

\section{Echinomma leptodermum Jörgensen}

Echinomma leptodermum Jörgensen, 1905, p. 116, pl. 8, fig. 33. Abundance: Few to rare.

Occurrence: Pleistocene, Saturnalis circularis Zone (Site 324).

\section{Echinomma popofskii Petrushevskaya}

Echinomma popofskii Petrushevskaya, 1968, p. 20-22, fig. 12, I-III; Chen, 1975, p. 505, pl. 20, fig. 13.

Abundance: Rare.

Occurrence: Pleistocene (Site 324).

\section{Genus PRUNOPYLE Dreyer}

Prunopyle Dreyer, 1889, p. 3.

\section{Prunopyle antarctica Dreyer}

Prunopyle antarctica Dreyer, 1889, p. 24-25, fig. 75; Riedel, 1958, p. 225 , pl. 1, fig. 7,8 ; Chen, 1975 , p. 511 , pl. 23 , fig. 5 , 6 .

Cromyechinus antarctica (Dreyer), Petrushevskaya, 1968, p. 22-27, fig. 13, I-VI; 14, I-VII.

Abundance: Rare to few.

Occurrence: Pleistocene and Pliocene. 
TABLE 2

\begin{tabular}{|c|c|c|c|c|c|c|c|c|c|c|c|c|c|c|c|c|c|c|c|c|c|c|c|c|c|c|c|c|c|c|c|c|c|c|c|c|c|c|c|c|}
\hline $\begin{array}{c}\text { Sample } \\
\text { (Interval in } \mathrm{cm} \text { ) }\end{array}$ & 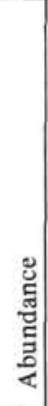 & 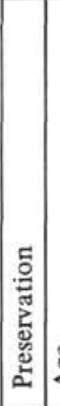 & 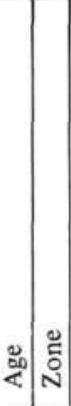 & 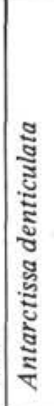 & 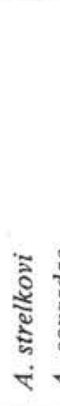 & 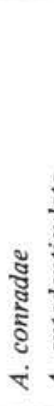 & 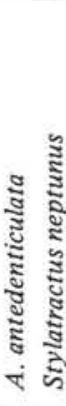 & & 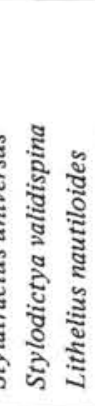 & 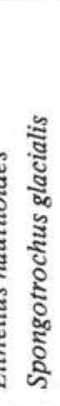 & 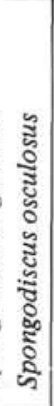 & 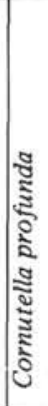 & 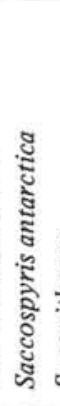 & 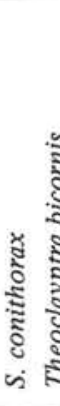 & 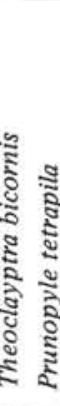 & $\frac{3}{3}$ & & 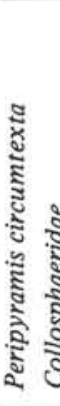 & 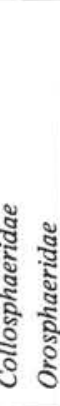 & 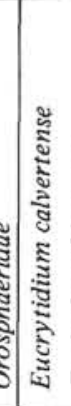 & 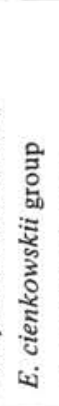 & 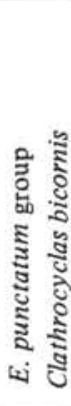 & 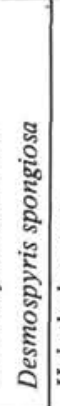 & 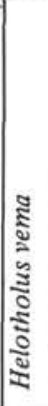 & 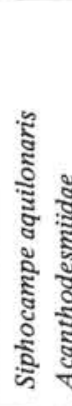 & 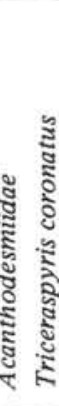 & 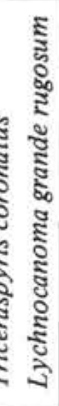 & 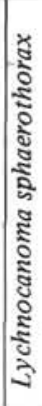 & 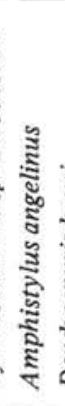 & & 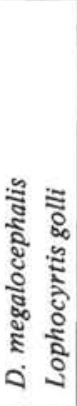 & 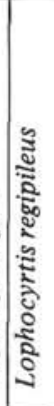 & 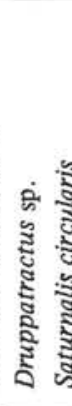 & & 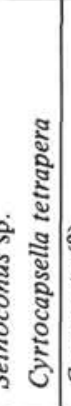 & 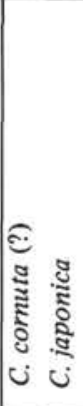 & 证 & 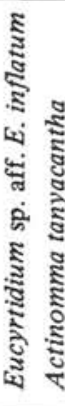 & 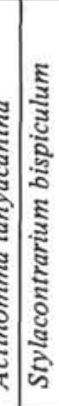 & 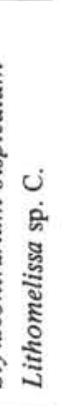 \\
\hline $323-1-1,74-76$ & - & - & & & & & & & & & & & & & & & & & & en & & & & & & & & & & & & & & & & & & & & \\
\hline & $\mathrm{R}$ & & & R & $\ldots$ & - & $-F$ & & & & & & & & & & & & & & & & & & & & & & & & & & & & & & & & & \\
\hline & $\mathrm{R}$ & & & - & - & & - & - & -- & - & & - & - & - & - - & - & - & - & - & & & & & & & & & & & & & & & & & & & & & \\
\hline $3-1-2,57-59$ & F & & & - & - & & $-R$ & - & $-\ldots$ & -- & & - & - & - & - & - & - & - & - & $-1-$ & - & - & - & - & - & - - & $-\ldots$ & & & & & & & & & & & & & \\
\hline $3-1-2,107-109$ & $c$ & & & C & C & - & $+R$ & - & - $R R$ & C & & R & $\mathrm{R}$ & - & - & + & - & - & - & $F$ & - & $-R$ & & $\mathrm{R}$ & $\ldots$ & - & $-\mathrm{R}$ & & & & & & & & & & & & & \\
\hline $3-1-2,139-141$ & $\mathrm{~F}$ & & & R & - & - & $-R$ & & R R & $\mathrm{R}$ & & - & - & - & - & - & - & - & - & $R$ & - & $-\mathrm{R}$ & $\mathrm{R}$ & $R$ & - & - & -- & & & & & & & & & & & & & \\
\hline & $\mathrm{F}$ & & & F & $\mathrm{R}$ & - & - & - & $-\quad-R$ & $\mathrm{~F}$ & $\mathbf{R}$ & $\mathrm{R}$ & $\mathrm{R}$ & $-R$ & R - & + & - & - & - & $-R$ & - & - & $\mathrm{R}$ & $\mathrm{R}$ & - & - - & -- & & & & & & & & & & & & & \\
\hline & $\mathrm{R}$ & & & $\mathrm{R}$ & $\mathrm{R}$ & - & -- & & - $\mathrm{R}-$ & - & - & - & $\mathrm{R}$ & - & - & - & - & - & - &.-- & - & - & -1 & + & - & - & -- & & & & & & & & & & & & & \\
\hline 3 & $\mathrm{R}$ & & & - & - & - & $-R$ & - & $-\ldots$ & - & - & - & - & - & - - & - & - & -1 & - - & $-1-$ & - & - - & & - & - - & - - & -- & & & & & & & & & & & & & \\
\hline 106 & $\mathrm{R}$ & 1 & & - & - & - & & & -- & -- & & - & - & - & - & - & - & - & - & $-1 R$ & - & - & - & - & $\ldots$ & - & -- & & & & & & & & & & & & & \\
\hline 18 & - & & & & & & & & & & & & & & & & & & & Barren & & & & & & & & & & & & & & & & & & & & \\
\hline 3 & $\mathrm{R}$ & & & - & - & - & $-\mathrm{R}$ & - & -- & $-R$ & - & - & - & - & $-\mathrm{R}$ & - & - & - & - - & $-R$ & - & - - & $\mathrm{R}$ & - & - & - & -- & & & & & & & & & & & & & \\
\hline 133 & $\mathrm{~F}$ & & & $\mathrm{R}$ & $\mathrm{R}$ & - & $-F$ & - & -- & $-\mathrm{C}$ & 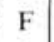 & . & - & - & - R & - & - & - & -- & - & - & - & - & - & - & - & -- & & & & & & & & & & & & & \\
\hline & C & & & $R$ & $\mathrm{R}$ & - & $-F$ & $\mathbf{R}$ & $-R$ & $\mathrm{~F}$ & & - & - & - & $-\mathrm{R}$ & + & - & - & - & - & - & $-R$ & & - & - & -- & -- & & & & & & & & & & & & & \\
\hline & $\mathrm{F}$ & & & - & - & - & $-F$ & - & -- & $-F$ & & - & - & - & $-F$ & + & -1 & $\mathrm{R}$ & - - & $-C$ & - & - + & & - & - & - - & -- & & & & & & & & & & & & & \\
\hline & R & & & - & - & - & - & - & $-\ldots$ & $-F$ & 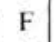 & - & - & - & - & - & - & - & - & C & - & - & & - & - & - & -- & & & & & & & & & & & & & \\
\hline 9-111 & I & & & - & - & - & $-R$ & & -- & $-F$ & & - & - & - & $-R$ & - & - & - & - & $-C$ & - & $-R$ & & - & - & - & -- & & & & & & & & & & & & & \\
\hline 3 & $\mathrm{~F}$ & & & - & $\mathrm{R}$ & - & $-F$ & - & -- & $-F$ & $\mathrm{~F}$ & - & - & - & - - & - & -1 & $\mathrm{R}$ & - & - C & - & -- & $\mathrm{R}$ & - & - & - & & & & & & & & & & & & & & \\
\hline & A & & & C & C 1 & R & R F & $\mathrm{F}$ & :- - & - & - & $\mathrm{F}$ & C & $\mathrm{R}-$ & $-F$ & C & -1 & $\mathrm{R}$ & -- & - A & - & $-\mathrm{A}$ & C & $\mathrm{F}$ & R C & C - & - & & & & & & & & & & & & & \\
\hline & $\mathrm{F}$ & & & - & - & - & - - & - & -- & $-F$ & & - & - & - & - & $R$ & - & - & - & $F$ & - & - & - & - & - & - R & $R$ & & & & & & & & & & & & & \\
\hline & 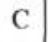 & 1 & & R & R F & R 1 & $\mathrm{~F} F$ & $\mathrm{R}$ & $-R$ & $\mathrm{C}$ & $\mathrm{F}$ & 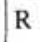 & 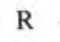 & - & - $\mathrm{R}$ & $\mathrm{F}$ & - & $-R$ & $\mathrm{R}$ - & C & - & $-R$ & - & - & - & $-\mathrm{C}$ & $\therefore \mathrm{C}$ & & & $\mathrm{R}$ & & & - & - & --1 & - - & $-\cdots$ & & & \\
\hline & C & c & & R & F $\mathrm{F}$ & F $\mathrm{I}$ & $\mathrm{F}-$ & $R$ & $-R$ & $\mathrm{~F}$ & - & R & $\mathrm{R}$ & $-R$ & $\mathrm{R} \quad \mathrm{R}$ & - & R & $\mathrm{R} R$ & R - & - $R$ & - & -- & - & - & - & $-R$ & $\mathrm{C}$ & - & - & - & -- & & $\mathrm{F} \quad \mathrm{R}$ & R - & -- & - - & $-\quad-R$ & & & 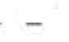 \\
\hline & $\mathrm{F}$ & 1 & & - & $-\mathrm{F}$ & $\mathrm{R}$ & - & - & -- & $R$ & - & - & - & - & - & - & $\mathrm{R}$ & - & - & $1-$ & - & - & - & - & - & -- & -- & R & - & - & -- & - & - & - & --1 & -- & $-\quad-R$ & $\mathrm{R}$ & & \\
\hline & - & & & & & & & & & & & & & & & & & & B & Barren & & & & & & & & & & & & & & & & & & & & \\
\hline & $\mathrm{F}$ & & & - & - & - & - & - & $-\cdots$ & - & - & - & - & - & -- & - & $\mathrm{F}$ & $-R$ & $\mathrm{R}$ - & - & - & - & - & - & - & - & -- & R & - & - & --1 & & - & - & -- & -- & -- & - & & \\
\hline & R & & & - & & - & - & - & --- & -- & - & - & - & - & - & - & - & - & $-F$ & $F$ & - & -- & - & - & - & - & -- & & - & -- & -- & & - & & --1 & -- & & & & \\
\hline 3 & F & 1 & & - & - & - & - & - & $\ldots-$ & -- & - & - & - & - & - & - & C & - & $-\mathrm{C}$ & C) - & - & -- & & - & - & - & -- & & - & - & -- & - & - & - & --1 & - - & $-\ldots$ & - & & \\
\hline & C & c & & - & $-c$ & C & - & - & -- & -- & - & F & - & $-\mathrm{R}$ & $\mathrm{R}$ - & - & C & $-F$ & F C & - & $\mathrm{F}$ & - & - & - & - & - & -- & & $\mathrm{R}$ & $-F$ & $\mathrm{~F} \quad-$ & - & - & $-\mathrm{R}$ & & F $\quad R$ & $\mathrm{R}$ & $-F$ & & $R$ \\
\hline & - & & & & & & & & & & & & & & & & & & & Barren & & & & & & & & & & & & & & & & & & & & \\
\hline $34-137$ & $\mathrm{~F}$ & M & & - & $=$ & - & $-\cdot$ & - & -- & -- & - & 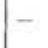 & - & - & -- & - & - & - & $-\mathrm{C}$ & $\mathrm{Cl}$ - & - & - & & - & - & - & -- & & 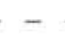 & - & -- & - & - & - & -- & -- & -- & - & & \\
\hline $142-144$ & - & & & & & & & & & & & & & & & & & & & Barren & & & & & & & & & & & & & & & & & & & & \\
\hline & $\mathrm{R}$ & 1 & & - & - & - & 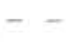 & & $-\ldots$ & -- & $=$ & & - & - & - - & - & $\mathrm{F}$ & - & $-\mathrm{C}$ & Cl- & - & - & - & - & - & - & -- & & $R$ & - & -- & & - & - & $-\mathrm{R}$ & $-R$ & $2-$ & - & & \\
\hline & $\mathrm{F}$ & M & & - & - & - & - & & -- & -- & - & - & - & - & - & - & $\mathrm{R}$ & $-R$ & R F & $\mathrm{F} \mid$ - & $\mathrm{R}$ & - & & - & - & - & -- & & - & - & -- & & - & - & $-\mathrm{R}$ & -- & -- & - & & \\
\hline & -1 & & & & & & & & & & & & & & & & & & & Barren & & & & & & & & & & & & & & & & & & & & \\
\hline & $\mathrm{F}$ & 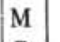 & & - & - & - & - & & --- & -- & - & & - & - & - & - & - & - & $-F$ & $F_{1}-$ & - & -- & - & - & - & -- & -- & & - & - & -- & - & - & - & -- & -- & $-\cdots$ & -- & & \\
\hline 10 & c & G & & - & - & - & -- & & -- & -- & - & - & - & - & -- & - & C & $-c$ & C C & c) - & C & $\mathrm{F}-$ & & - & - & -- & -- & & $R$ & $R+$ & $+F$ & C & - & $-R$ & $\begin{array}{ll}R & R\end{array}$ & -- & $-F-$ & - & & \\
\hline$\left(\mathrm{G}_{\mathrm{B}}\right)$ & R & P & & - & - & - & - & & -- & -- & - & - & - & - & - & - & $\mathrm{R}$ & - & - & - & - & - & & - & - & -- & -- & - & - & - & $-\mathrm{R}$ & - & - & - & --1 & - - & -- & - & & - \\
\hline & $\mathrm{F}$ & c & & - & - & - & -- & & -- & -- & - & & - & - & - & & - & - & $-F$ & $\mathrm{~F} \mid-$ & - & -- & & - & - & - & -- & & - & - & -- & & - & - & -- & -- & -- & - & & 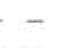 \\
\hline & $\begin{array}{l}\mathrm{R} \\
\mathrm{R}\end{array}$ & G & & - & - & - & - & & -- & - & -1 & & - & - & - & & - & - & - $\mathbf{R}$ & R | - & - & - & & - & - & -- & -- & & - & -- & --1 & - & - & - & --1 & -- & -- & $\pi$ & & \\
\hline 323-7-1 (Dk. grees & & & & & & & & & & & & & & & & & & & & & & & & & & & & & & & & & & & & & & & & \\
\hline
\end{tabular}


323-7-3, 19-21 323-7, CC

323-8-1, 34-36

323-8-1, 40-42

323-8-1, 98-100

323-8, CC

323-9, CC

323-10-1, 96-98

323-10-2, 103-104

323-10-3, 67-68

$323-10$, CC

323-11-1, 36-38

323-11-1, 130-132

23-11-2, 66-68

$323-12-1,129-131$

323-12-2, 14-16

323-12-2, 74-76

323-12, CC

323-13-1, 104-106

$323-13-5,83-84$

323-13-6, 31-32

323-13-6, 108-110

323-13, CC

323-14-2, 69-71

323-14-2, 122-124

323-14, CC

323-15-1, 110-112

323-15-2, 105-107

323-15-3, 116-118

323-15-4, 24-26

323-15-6, 18-20

323-15, CC

323-16-1, 37-39

323-16-1, 94-96

323-16-2, 40-42

323-16-2, 90-92

323-16-3, 90-92

323-16-4, 57-59

323-16, CC

323-17-1, 81-83

$323-17$, CC

323-18-2, 138-140

323-18-3, 63-65

323-18-3, 127-129

323-18-3, 143-14

323-18-4, 58-60

323-18-4, 85-87

323-18-4, 105-107

323-18-5, 0-2

323-18-5, 47-49

323-18-5, 138-140

323-18-6, (Basalt

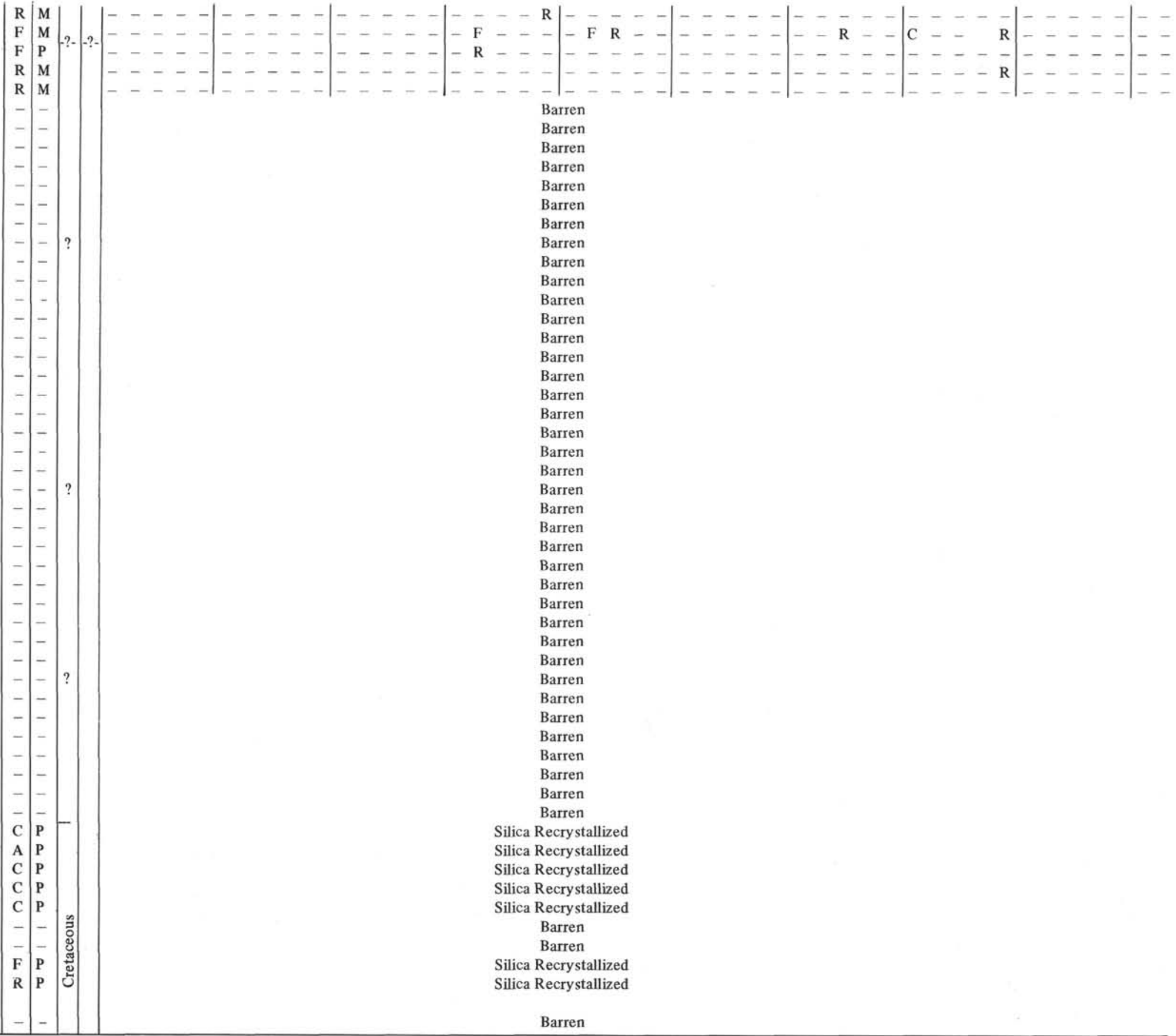


TABLE 3

Radiolarians at Site 324

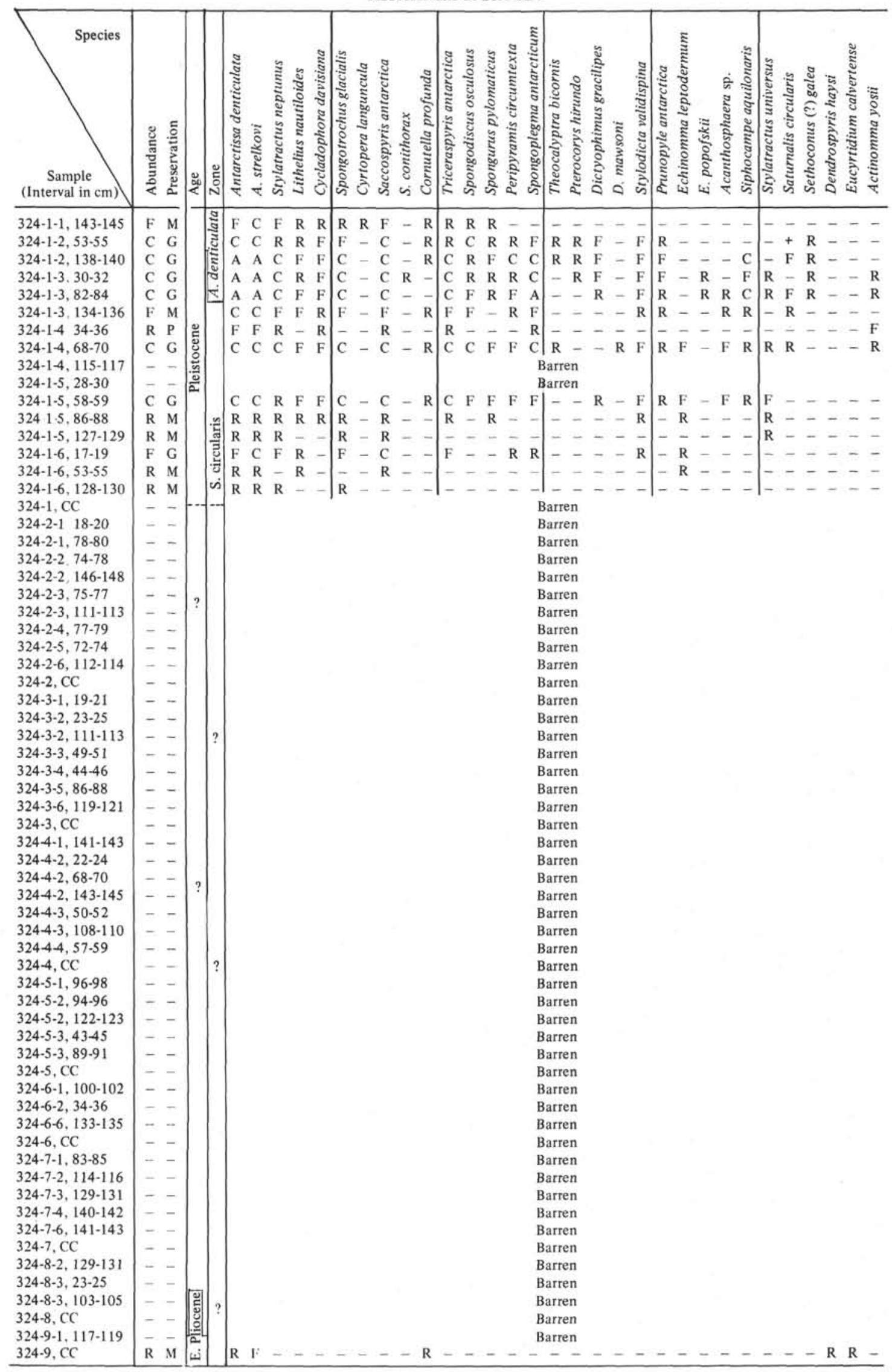


TABLE 4

Radiolarians at Site 325

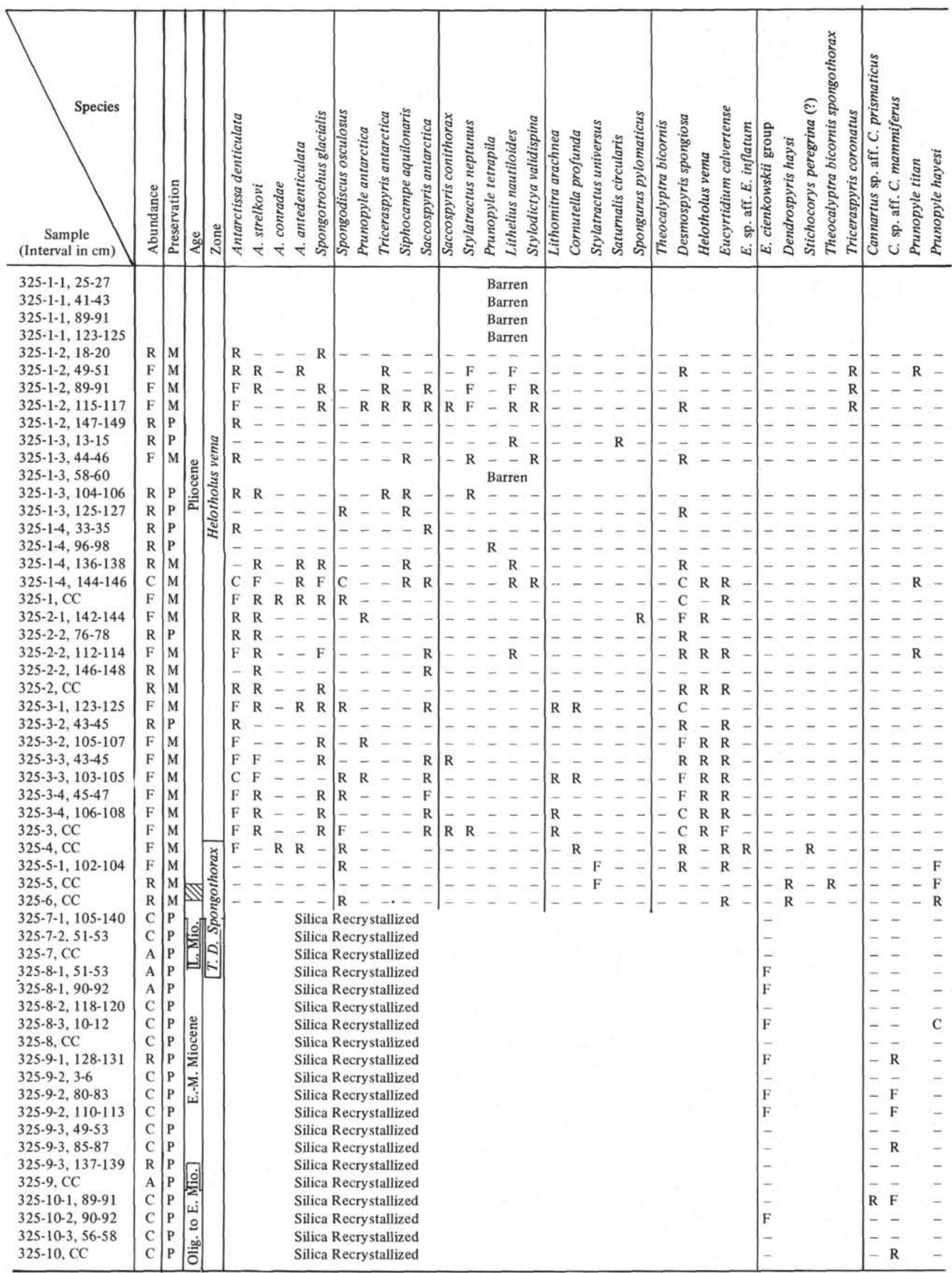


TABLE 5

Taxonomic Designations as Given by Chen (1975), and Followed in this Report, and Equivalent Designations of Petrushevskaya (1975)

\begin{tabular}{|c|c|}
\hline Chen, Leg 28 & Petrushevskaya, Leg 29 \\
\hline Taxa & Equivalent Taxa \\
\hline $\begin{array}{l}\text { Amphistylus angelinus-Plate } 21 \text {, fig. } 3,4 . \\
\text { Prunopyle titan-Plate } 23 \text {, fig. } 1,2 . \\
\text { Prunopyle hayesi-Plate } 9 \text {, fig. } 3-5 \text {. } \\
\text { Cyrtocapsella isopera-Plate } 11 \text {, fig. } 7-9 \text {. } \\
\text { Dendrospyris haysi-Plate } 15 \text {, fig. } 3,4 \text {. } \\
\text { Spongomelissa dilli-Plate } 13 \text {, fig. } 6,7 \text {. } \\
\text { Antarctissa denticulata-Plate } 18 \text {, fig. } 5,6 \text {. } \\
\text { Antarctissa antedenticulata-Plate } 18, \text { fig. } 1,2 \text {. } \\
\text { Antarctissa antedenticulata-Plate } 18 \text {, fig. } 1,2 \text {. } \\
\text { Antarctissa conradae-Plate } 17 \text {, fig. } 1-5 \text {. } \\
\text { Theocalyptra bicornis spongothorax-Plate } 12 \text {, fig. } 1-3 \text {. } \\
\text { Lophocyrtis regipileus-Plate } 12 \text {, fig. } 6,7 \text {. } \\
\text { Cyclampterium (?) longiventer-Plate } 10 \text {, fig. } 7 \text {. }\end{array}$ & $\begin{array}{l}\text { Stylosphaera hispida group-Plate 2, fig. } 4 \text {. } \\
\text { Lithocarpium titan-Plate } 4 \text {, fig. } 3 \text {. } \\
\text { Ommatodiscus haeckeli group-Plate } 3 \text {, fig. } 12-16 \text {. } \\
\text { Theocorys longithorax-Plate } 8 \text {, fig. } 17,18 \text {. } \\
\text { Desmospyris rhodospyroides-Plate } 10 \text {, fig. } 31,32 \text {. } \\
\text { Corythomelissa horrida-Plate } 11 \text {, fig. } 14,15 \text {. } \\
\text { Antarctissa cylindrica-Plate } 11 \text {, fig. } 19,20 \text {. } \\
\text { Antarctissa robusta-Plate } 11 \text {, fig. } 21,22 \text {. } \\
\text { Antarctissa capitata-Plate } 11 \text {, fig. } 24 \text {. } \\
\text { Botryopera deflandrei-Plate } 11 \text {, fig. } 30-32 \text {. } \\
\text { Clathrocyclas cabrilloensis group-Plate } 15 \text {, fig. } 27-29 \text {. } \\
\text { Clathrocyclas titanothericeraos-Plate } 23 \text {, fig. } 1 \text {. } \\
\text { Thyrsocyrtis sp.-Plate } 8 \text {, fig. } 10 \text {. }\end{array}$ \\
\hline
\end{tabular}

Prunopyle hayesi Chen

(Plate 7, Figures 1-3)

Prunopyle hayesi Chen, 1975, p. 483, pl. 9, fig. 3-5.

Abundance: Few to common.

Occurrence: Miocene and early Pliocene.

\section{Prunopyle tetrapila Hays}

Prunopyle tetrapila Hays, 1965, p. 172, pl. II, fig. 5.

Cromyechinus tetrapila (Hays), Petrushevskaya, 1972, p. 575, pl. 9, fig. 11.

Abundance: Rare to few.

Occurrence: Pliocene (Sites 323 and 325).

\section{Prunopyle titan (?) Campbell and Clark (Plate 7, Figure 6)}

Prunopyle titan Campbell and Clark, 1944, p. 20, pl. 3, fig. 1-3; Hays, 1965 , p. 173, pl. II, fig. 4; Bandy et al., 1971, p. 9, pl. 1, fig. 6-10; Keany and Kennett, 1972, p. 539, fig. 4.

Abundance: Few to common.

Occurrence: Pliocene.

Remarks: The Prunopyle titan reported from Antarctic sediments by numerous authors is not believed to be the same species that was originally described by Campbell and Clark (1944) from Miocene sediments in California. Campbell and Clark (1944) clearly state that $P$. titan does not have any internal medullary shell. The Antarctic form referred to as $P$. titan, on the other hand, does have a loosely packed internal structure composed of at least four spirals. Although some of the Antarctic specimens do appear to be hollow, a preliminary SEM investigation indicates that this is a result of differential dissolution. The internal spirals are more easily dissolved with respect to the cortical prune-shaped outer shell. Specimens in all states of preservation have been observed and the preferential destruction of the internal spirals observed (Weaver, in preparation.)

\section{Genus STYLATRACTUS Haeckel}

Stylatractus Haeckel, 1887, p. 328.

\section{Stylatractus neptunus Haeckel}

Stylatractus neptunus Haeckel, 1887, p. 328, pl. 17, fig. 6 .

Stylatractus neptunus Haeckel (?) Riedel, 1958, p. 226, pl. 1, fig. 9. Stylatractus sp. Petrushevskaya, 1968, p. 27-28, fig. 15, I-IV; 32, III. Abundance: Rare to few.

Occurrence: Pliocene and Pleistocene.

Stylatractus universus Hays

(Plate 5, Figures 3, 6; Plate 6, Figures 5-7)

Stylatractus sp. Hays, 1965, p. 167, pl. 1, fig. 6.

Stylatractus universus Hays, 1970, p. 215, pl. 1, fig. 1, 2; Kling, 1971, p. 1086 , pl. 1, fig. 6 ; Chen, 1975 , p. 507 , pl. 21, fig. 5-9. Abundance: Rare to few.

Occurrence: Pliocene? and Pleistocene.
Remarks: The specimens identified as $S$. universus by Chen (1975) and in this study from early Pliocene sediments may be a totally different species. In contrast to the late Pleistocene form of $S$. universus, the "Pliocene form" has: (1) narrower polar spines, (2) a cortical shell that is less elliptical and elongated along the polar axis, (3) apparently only one inner medullary shell (this would place this species into the genus Lithatractus), and (4) no supporting spines that protrude from the cortical shell. The latest occurrence of this "Pliocene form" of $S$. universus is within the Gilbert magnetic epoch and is coincident with the top of the $T . b$. spongothorax Zone of Chen (1975).

\section{Genus SPONGOPLEGMA Haeckel}

Spongoplegma Haeckel, 1881, p. 455.

\section{Spongoplegma antarcticum Haeckel}

Spongoplegma antarcticum Haeckel, 1887, p. 90; Hays, 1965, p. $165-$ 167 , pl. 1, fig. 1; Chen, 1975, p. 509, pl. 22, fig. $3,4$. Diploplegma banzare Riedel, 1958, p. 223, pl. 1, fig. 3, 4. Cenosphaera antarctica Nakaseko, 1959, p. 5, pl. 1, fig. 3-6.

Diploplegma aquatica (Popofsky), Petrushevskaya, 1968, p. 14-18, fig. 9, I-III; 10, I-VI.

Abundance: Rare to common.

Occurrence: Pleistocene.

\section{Genus SPONGURUS Haeckel}

Spongurus Haeckel, 1860.

\section{Spongurus pylomaticus Riedel}

Spongurus pylomaticus Riedel, 1958, p. 226, pl. 1, fig. 10, 11; Petrushevskaya, 1968 , p. 32, fig. 16, I-II.

Abundance: Rare to few.

Occurrence: Pliocene and Pleistocene (Sites 324 and 325).

\section{Genus STYLACONTARIUM Popofsky}

Stylacontarium Popofsky, 1912, p. 90.

\section{Stylacontarium bispiculum Popofsky}

Stylacontarium bispiculum Popofsky, 1912, p. 91, pl. 2, fig. 2; Chen, 1975 , p. 507 , pl. 21 , fig. $1,2$.

Abundance: Rare.

Occurrence: Middle Miocene (Site 323).

\section{Family COLLOSPHAERIDAE Müller, 1858}

Members of this family were identified in early Pliocene and Miocene sediments. Specimens encountered are similar to those illustrated by Bandy et al., 1971, p. 11, pl. 2, fig. 8, 9; and Kennett and Brunner, 1973, p. 2045, fig. 2, A, B. 


\section{Family OROSPHAERIDAE Haeckel, 1887}

\section{Genus OROSCENA Haeckel}

Oroscena Haeckel 1887, emend. Friend and Riedel, 1967.

\author{
Oroscena sp. \\ (Plate 3, Figure 11)
}

Oroscena sp. Bandy et al., 1971, p. 11, pl. 2, fig. 2, 3.

Abundance: Rare to common.

Occurrence: Miocene.

Remarks: Only digitate spines are illustrated. The orosphaerid spines are similar to those of Oroscena spp. Friend and Riedel, 1967, p. 229, pl. 2, fig. 5, an orosphaerid which occurs in assemblages from middle Miocene tropical Pacific sediments.

\section{Family LITHELIIDAE Haeckel}

Litheliidae Haeckel, 1862, p. 515.

\section{Genus LITHELIUS Haeckel}

Lithelius Haeckel, 1860, p. 843; Sanfilippo and Riedel, 1973, p. 522.

\section{Lithelius nautiloides Popofsky}

Lithelius nautiloides Popofsky, 1908, p. 230-231, pl. XXVII, fig. 4; Riedel, 1958, p. 228-229, pl. 2, fig. 3; Petrushevskaya, 1968, p. 5052 , fig. 27-28; Chen, 1975 , p. 513 , pl. 24 , fig. 7.

Abundance: Few to rare.

Occurrence: Miocene to Pleistocene.

\section{Family SPONGODISCIDAE Haeckel}

Spongodiscidae Haeckel, 1862, emend. Riedel, 1967b, p. 295.

\section{Genus SPONGODISCUS Ehrenberg}

Spongodiscus Ehrenberg, 1854, p. 237; Sanfilippo and Riedel 1973, p. 524 .

\section{Spongodiscus osculosus (Dreyer)}

Spongopyle osculosa Dreyer, 1889, p. 42-43, pl. VI, fig. 99, 100; Riedel, 1958, p. 226-227, pl. 1, fig. 12.

Spongodiscus osculosus (Dreyer), Petrushevskaya, 1968, p. 39-40, fig. 20, I, II; Chen, 1975, p. 513, pl. 24, fig. 4.

Abundance: Rare to common.

Occurrence: Miocene through Pleistocene.

\section{Genus SPONGOTROCHUS Haeckel}

Spongotrochus Haeckel, 1860, p. 844

\section{Spongotrochus glacialis Popofsky}

Spongotrochus glacialis Popofsky, 1908, p. 228-229, pl. 26, fig. 7, 8; pl 27, fig. 1; Petrushevskaya, 1968, p. 40-50, fig. 21, I-VII; fig. 22, IVII; fig. 26, II; Chen, 1975 , p. 513 , pl. 24, fig. $5,6$.

Spongotrochus (?) glacialis Popofsky, Riedel, 1958, p. 227-228, pl. 2 , fig. 1, 2.

Abundance: Few to common.

Occurrence: Pliocene and Pleistocene.

\section{Genus STYLODICTYA Ehrenberg}

Stylodictya Ehrenberg 1847, emend. Kozlova, 1972, p. 525.

\section{Stylodictya validispina Jörgensen}

Stylodictya validispina Jörgensen, 1905 , p. 119 , pl. 10, fig. 40 ; Petrushevskaya, 1968 , p. 30-31, fig. 17, IV, V.

Abundance: Rare to few.

Occurrence: Pliocene and Pleistocene.

Subfamily ARTISCINAE Haeckel 1881, emend. Riedel 1967b

\section{Genus CANNARTUS Haeckel}

Cannartus Haeckel, 1881, p. 462, emend. Riedel, 1971, p. 652.

\section{Cannartus sp. aff. C. prismaticus (Haeckel)}

(Plate 5, Figure 9)

Cannartus sp. aff. C. prismaticus (Haeckel), Chen, 1975, p. 505, pl. 20 , fig. 7.

Abundance: Rare.

Occurrence: Oligocene (?) to early Miocene.

\section{Cannartus sp. aff. C. mammiferus (Haeckel)}

(Plate 5, Figures 7, 8)

Remarks: Rare to few specimens were found in Cores 9 and 10 at Site 325. All specimens were partially or totally recrystallized and vaguely resemble $C$. mammiferus on the basis of size, form, and the existence of remanent protuberances on the sides of the cortical shell (Plate 5, Figures 7, 8).

\section{Subfamily SATURNALINAE Deflandre, 1953}

\section{Genus SATURNALIS Haeckel}

Saturnalis Haeckel, 1881 , p. 450 , emend. Nigrini, 1967

\section{Saturnalis circularis Haeckel \\ (Plate 1, Figure 4)}

Saturnalis circularis Haeckel, 1887, p. 131; Nigrini, 1967, p. 25, pl. 1, fig. 9; Kling, 1973, p. 635, pl. 1, fig. 21-25; pl. 7, fig. 1-5; Chen, 1975 , p. 513 , pl. 24 , fig. 2.

Saturnalis planetes Haeckel, 1887, p. 142, pl. 16, fig. 7; Hays, 1965, p. 167, pl. 1 , fig. 5 .

Abundance: Rare to few.

Occurrence: Pliocene and Pleistocene.

\section{Suborder NASSELARIA Ehrenberg}

Family ACANTHODESMIIDAE Haeckel

Acanthodesmiidae Haeckel, 1862; emend. Riedel, 1967b, p. 296.

\section{Genus DENDROSPYRIS Haeckel}

Dendrospyris Haeckel, 1881, p. 44; emend. Goll, 1968, p. 1417.

\section{Dendrospyris haysi Chen}

(Plate 2, Figures 7-9; Plate 7, Figure 4)

Dendrospyris haysi Chen, 1975 , p. 495, pl. 15, fig. 3-5. Abundance: Rare to few.

Occurrence: Miocene and early Pliocene.

\section{Dendrospyris megalocephalis Chen}

(Plate 3, Figures 9, 10)

Dendrospyris megalocephalis Chen, 1975 , p. 493 , pl. 14, fig. 3-5. Abundance: Few.

Occurrence: Middle Miocene (Site 323).

$$
\text { Genus DESMOSPYRIS Haeckel }
$$

Desmospyris Haeckel, 1881 , p. 443; 1887, p. 1089

\section{Desmospyris spongiosa Hays}

(Plate 1, Figure 12)

Desmospyris spongiosa Hays, 1965, p. 173-175, pl. 2, fig. 1; Keany and Kennett, 1972, p. 539, fig. 4 , No. 12-13.

Abundance: Rare to common.

Occurrence: Pliocene.

\section{Genus TRICERASPYRIS Haeckel}

Triceraspyris Haeckel, 1881, p. 441.

\section{Triceraspyris antarctica (Haecker)} (Plate 1, Figures 2, 3)

Phormospyris antarctica Haecker, 1907, p. 124, fig. 9.

Triceraspyris antarctica (Haecker), Haecker, 1908, p. 445-446, pl. 84, fig. 586; Riedel, 1958, p. 230, pl. 2, fig. 6, 7; Petrushevskaya, 1964, p. $1121-1123$, fig. 1 .

Abundance: Rare to common.

Occurrence: Pliocene and Pleistocene. 
Triceraspyris coronatus Weaver n. sp.

(Plate 2, Figures 4, 5; Plate 6, Figures 8, 9)

Description: Shell, crown-shaped and heavily constructed. Sagittal ring D-shaped. Shell has deep-set circular to subcircular pores of uniform size arranged in longitudinal rows. Pores adjacent to the sagittal ring tend to be larger and elliptical in outline. Three basal feet slightly outwardly diverging, often bifurcated or trifurcated distally.

Discussion: A slight sagittal stricture is observed in some specimens. Well preserved specimens also have long, broad basal feet with a small amount of lattice-work between the proximal ends. Hays and Opdyke $(1967$, p. 9$)$ have previously referred to Triceraspyris coronatus as Triceraspyris sp.

Measurements: Based on 25 specimens from Samples 323-2-1, 132-134 $\mathrm{cm}$ and $\mathrm{E} 34-5,300 \mathrm{~cm}$ : Width of shell, 114-116 $\mu \mathrm{m}$; length of shell, 84-96 $\mu \mathrm{m}$; width of basal feet, 6-18 $\mu \mathrm{m}$; length of basal feet, 25 . $50 \mu \mathrm{m}$; pore diameter $8-15 \mu \mathrm{m}$.

Abundance: Common.

Occurrence: Latest Miocene and Pliocene, Southern Ocean.

Type locality: DSDP Site 323, Core 2, Section 1, 132-134 cm.

Repository: Holotype deposited at the U.S. National Museum, Washington, D.C.

\section{Family ARTOSTROBIIDAE Riedel}

Artostrobiidae Riedel, 1967a, p. 149

\section{Genus LITHOMITRA Bütschli}

Lithomitra, Butschli, 1882.

\section{Lithomitra arachnea (Ehrenberg)}

Eucyrtidium lineatum arachneum Ehrenberg, 1862, p. 299.

Lithomitra lineata (Ehrenberg) Haeckel, 1881, p. 1484.

Lithomitra arachnea (Ehrenberg) Riedel, 1958, p. 242-243, pl. 4, fig. 7, 8; Petrushevskaya, 1962, p. 339-340, fig. 9, 10; Ling et al., 1971 , p. 716 , pl. 2 , fig. 13,14

Abundance: Rare.

Occurrence: Pliocene.

\section{Genus SIPHOCAMPE Haecke}

Siphocampe Haeckel, 1887.

\section{Siphocampe aquilonaris (Bailey)}

Eucyrtidium aquilonaris Bailey, 1856, p. 4, pl. 1, fig. 9.

Lithocampe aquilonaris (Bailey), Haeckel, 1862, p. 317; 1887, p. 1504.

Lithocampe (?) aquilonaris (Bailey), Petrushevskaya, 1968, p. 140-141, fig. $79,1,11$.

Siphocampe aquilonaris (Bailey), Ling et al., 1971, p. 716, pl. 2, fig. 12.

Abundance: Rare to common.

Occurrence: Pliocene and Pleistocene.

\section{Family PLAGONIIDAE Haecekl}

Plagoniidae Haeckel, 1881, emend. Riedel, 1967b

Genus ANTARCTISSA Petrushevskaya

Antarctissa Petrushevskaya, 1968, p. 83.

\section{Antarctissa denticulata (Ehrenberg)}

Lithobotrys denticulata Ehrenberg, 1844, p. 203.

Lithopera denticulata (Ehrenberg), Ehrenberg, 1873, pl. 12, fig. 7; Haeckel, 1887, p. 1083; Haecker, 1907, p. 123-124, fig. 8.

Permomelissa denticulata (Ehrenberg) Haecker, 1908, p. 448-452, pl. 84 , fig. 582, 583, 591; Riedel, 1958 , p. 236 , pl. 3, fig. 9.

Helotholus histricosa var. clausa Popofsky, 1908, p. 281-282, pl. XXXIII, fig. 1 .

Antarctissa denticulata (Ehrenberg) Petrushevskaya, 1968, p. 84-86, fig. 49, I-IV.

Abundance: Rare to common.

Occurrence: Pliocene and Pleistocene.

\section{Antarctissa antedenticulata Chen}

Antarctissa antedenticulata Chen, 1975 , p. 501, pl. 18, fig. 1, 2 Abundance: Rare to few.

Occurrence: Earliest Pliocene.
Antaretissa conradae Chen

Antarctissa conradae Chen, 1975, p. 499, pl. 17, fig. 1-5.

Abundance: Rare to common.

Occurrence: Middle Miocene and Pliocene.

\section{Antarctissa strelkovi Petrushevskaya}

Helotholus histricosa Jörgensen, 1905, p. 137, pl. 16, fig. 86-88; Popofsky, 1908, p. 279-281, pl. 32, fig. 1-5; pl. 36, fig. 2; Riedel, 1958 , p. $234-235$, pl. 3 , fig. 8 .

Helotholus longus Popofsky, 1908, p. 282-283, pl. 34, fig. 2.

Antarctissa strelkovi Petrushevskaya, 1968, p. 88-90, fig. 51, III-VI; Chen, 1975, p. 499, pl. 17, fig. 6-9.

Abundance: Rare to abundant.

Occurrence: Pliocene and Pleistocene.

\section{Genus HELOTHOLUS Jörgensen}

Helotholus Jörgensen, 1905, p. 137.

\section{Helotholus vema Hays}

(Plate 1, Figures 10, 11)

Helotholus vema Hays, 1965, p. 176, pl. 2, fig. 3; Chen, 1975, p. 497, pl. 16, fig. 1-4.

Pseudocubus vema (Hays), Keany and Kennett, 1972, p. 539, fig. 4, no. 10,11

Abundance: Rare to few.

Occurrence: Pliocene.

\section{Genus LITHOMELISSA Ehrenberg}

Lithomelissa Ehrenberg, 1847b, p. 54.

Lithomelissa sp. C Chen

(Plate 8, Figures 2, 3)

Lithomelissa sp. C Chen, 1975, p. 487, pl. 11, fig. 3, 4

Abundance: Rare.

Occurrence: Miocene.

Family PTEROCORYIDAE Haeckel

Pterocoryidae Haeckel, 1881, emend, Riedel, 1967b.

\section{Genus PTEROCORYS Haeckel}

Pterocorys Haeckel, 1881, p. 435.

\section{Pterocorys hirundo Haecke}

Pterocorys hirundo Haeckel, 1887, p. 1318, pl. 71, fig. 4; Riedel, 1958, p. 238 , pl. 4 , fig. 1 ; Chen, 1975 , p. 503 , pl. 19 , fig. 3 .

Pterocorys ((?) hirundo Haeckel, Petrushevskaya, 1968, p. 114-116, fig. $67, \mathrm{I}-\mathrm{V}$.

Abundance: Rare.

Occurrence: Pliocene and Pleistocene.

\section{Family THEOPERIDAE Haeckel}

Theoperidae Haeckel, 1881, emend. Riedel, 1967b, p. 296.

Genus CLATHROCYCLAS Haeckel

Clathrocyclas Haeckel, 1882, p. 434.

\section{Clathrocyclas bicornis Hays}

Clathrocyclas bicornis Hays, 1965, p. 179, pl. 3, fig. 3; Chen, 1975, p. 489 , pl. 12 , fig. 8,9 .

Abundance: Rare to abundant.

Occurrence: Pliocene.

\section{Genus CORNUTELLA Ehrenberg}

Cormutella Ehrenberg, 1838, p. 128.

\section{Cornutella profunda Ehrenberg}

Cormutella clathrata B. profunda Ehrenberg, 1858, pl. 35b., fig. 21; Bailey, 1856, p. 2, pl. 1, fig. 23

Cormutella profunda Ehrenberg, 1858, p. 31; Riedel, 1958, p. 232 . pl. 3, fig. 1, 2.

Cormutella hexagona Haeckel, 1887, p. 1180, pl. 54, fig. 9. 
Abundance: Rare to few.

Occurrence: Pliocene and Pleistocene.

\section{Genus CYCLADOPHORA Ehrenberg}

Cycladophora Ehrenberg, 1847b, p. 54.

\section{Cycladophora davisiana Ehrenberg}

(Plate 1, Figure 7)

Cycladophora (?) davisiana Ehrenberg, 1862, p. 297; 1873, pl. 2, fig. 11.

Theocalpytra davisiana (Ehrenberg), Riedel, 1958, p. 239, pl. 4, fig. 2, 3.

Cycladophora davisiana Ehrenberg, Petrushevskaya, 1968, p. 120-122, fig. 69, I-VII; Chen, 1975, p. 491, pl. 13, fig. 3.

Abundance: Rare to few.

Occurrence: Pleistocene.

\section{Genus CYRTOCAPSELLA Haeckel}

Cyrtocapsella Haeckel, 1887; Sanfilippo and Riedel, 1970, p. 451; Riedel and Sanfilippo, 1970, p. 530.

Cyrtocapsella isopera Chen

(Plate 3, Figures 5, 6; Plate 9, Figures 4, 6)

Cyrtocapsella isopera Chen, 1975, p. 467, pl. 11, fig. 7-9.

Abundance: Rare to few.

Occurrence: Middle Miocene.

\section{Cyrtocapsella tetrapera Haeckel}

(Plate 3, Figures 1-3; Plate 9, Figures 1, 2, 3, 7)

Cyrtocapsa (Cyrtocapsella) tetrapera Haeckel, 1887, p. 1512, pl. 78, fig. 5 .

Cyrtocapsella tetrapera Haeckel, Sanfilippo and Riedel, 1970, p. 453, pl. 1, fig. 16-18; Chen, 1975, p. 505, pl. 20, fig. 1.

Abundance: Rare to abundant.

Occurrence: Middle Miocene.

\section{Cyrtocapsella cornuta (?) Haecke}

(Plate 3, Figure 8)

Cyrtocapsa (Cyrtocapsella) cornuta Haeckel, 1887, p. 1513, pl. 78, fig. 9.

Cyrtocapsella cornuta Haeckel, Sanfilippo and Riedel, 1970, p. 453, pl. 1, fig. 19-20; Kling, 1973, p. 636, pl. 11, fig. 16-18.

Abundance: Few.

Occurrence: Middle Miocene.

\section{Cyrtocapsella japonica (?) (Nakaseko)}

(Plate 3, Figure 7)

Eusyringium japonicum Nakaseko, 1963, p. 193, pl. 4, fig. 1-3.

Cyrtocapsella japonica (Nakaseko), Sanfilippo and Riedel, 1970, p. 452, pl. 1, fig. 13-15; Kling, 1973, p. 636, pl. 11, fig. 19, 20. Abundance: Rare.

Occurrence: Middle Miocene.

\section{Genus CYRTOPERA Haeckel}

Cyrtopera Haeckel, 1881, p. 439

\section{Cyrtopera languncula Haecke}

Stichopera pectinata Haeckel, 1887, p. 1449, pl. 75, fig. 11; Kling, 1973 , p. 638 , pl. 3 , fig. $25-27$, pl. 10 , fig. $1-5$.

Cyrtopera languncula Haeckel, 1887, p. 1451, pl. 75, fig. 10; Chen, 1975 , p. 501 , pl. 18 , fig. 9.

Abundance: Rare.

Occurrence: Pliocene and Pleistocene.

\section{Genus DICTYOPHIMUS Ehrenberg}

Dictyophimus Ehrenberg, 1847b, p. 53.

\section{Dictyophimus mawsoni Riedel}

Dictyophimus mawsoni Riedel, 1958, p. 234, pl. 3, fig. 6, 7; Chen, 1975 , p. 503 , pl. 19 , fig. 2

Dictyophimus (?) mawsoni Riedel, Petrushevskaya, 1968, p. 73-74, fig. 43

Abundance: Rare.

Occurrence: Pleistocene.
Dictyophimus gracilipes Bailey

Dictyophimus gracilipes Bailey, 1856, p. 4, pl. 1, fig. 8; Popofsky, 1908 , p. $274-275$, pl. 31 , fig. 15 (in part); Riedel, 1958, p. 233-234, pl. 3, fig. 5; Petrushevskaya, 1968, p. 65-67, fig. 38, I-VII; fig. 39 , I-III.

Abundance: Few.

Occurrence: Pleistocene.

\section{Genus EUCYRTIDIUM Ehrenberg}

Eucyrtidium Ehrenberg, 1847b, p. 43

\section{Eucyrtidium calvertense Martin}

(Plate 1, Figure 9)

Eucyrtidium calvertense Martin, 1904, p. 450, pl. 130, fig. 5; Hays, 1965 , p. 181 , pl. 3, fig. 4; 1970, p. 213, pl. 1, fig. 6; Kling, 1973 , p. 636 , pl. 4 , figs. $16,18,19$; Chen, 1975 , p. 495 , pl. 15 , fig. 9. Abundance: Rare to common.

Occurrence: Miocene and Pliocene.

Eucyrtidium cienkowskii Haeckel group (Plate 4, Figures 3-5; Plate 8, Figures 7-9)

cf. Eucyrtidium cienkowskii Haeckel, 1887, p. 1493, pl. 80, fig. 9.

cf. Eucyrtidium cienkowskii Haeckel group, Sanfilippo et al., 1973 p. 221 , pl. 5, fig. $7-11$; Chen, 1975, p. 495 , pl. 15, fig. 7.

Abundance: Rare to common.

Occurrence: Miocene.

\section{Eucyrtidium sp. aff. E. inflatum Kling} (Plate 2, Figure 12)

Eucyrtidium inflatum Kling, 1973, p. 636, pl. 11, fig. 7, 8. Abundance: Rare to few.

Occurrence: Latest Miocene and early Pliocene.

Eucyrtidium punctatum (Ehrenberg) group (Plate 4, Figures 1, 2; Plate 8, Figures 4-6)

cf. Lithocampe punctata Ehrenberg, 1844, p. 84.

cf. Eucyrtidium punctatum (Ehrenberg)-Ehrenberg, 1847, p. 43; Ehrenberg, 1854, pl. 22, fig. 24.

cf. Artostrobus zitteli Vinassa de Regny, 1900, p. 586, pl. 3, fig. 19.

Eucyrtidium punctatum (Ehrenberg) group, Chen, 1975, p. 495, pl. 15, fig. 8; Sanfilippo et al., 1973, p. 221, pl. 5, fig 15, 16.

Abundance: Rare to few.

Occurrence: Middle Miocene.

\section{Genus LOPHOCYRTIS Haeckel}

Lophocyrtis Haeckel, 1887, p. 1410.

\section{Lophocyrtis golli Chen}

(Plate 4, Figures 9, 10; Plate 9, Figures 8, 9)

Lophocyrtis golli Chen, 1975 , p. 489 , pl. 12, fig. 4, 5 . Abundance: Rare to few. Occurrence: Middle Miocene (Site 323).

Lophocyrtis regipileus Chen

(Plate 4, Figures 6-8; Plate 7, Figures 7-9)

Lophocyrtis regipileus Chen, 1975, p. 489, pl. 12, fig. 6, 7. Abundance: Common.

Occurrence: Middle Miocene (Site 323).

\section{Genus LYCHNOCANOMA Haeckel}

Lychnocanoma Haeckel, 1887, p. 1229.

\section{Lychnocanoma grande rugosum (Riedel)}

(Plate 9, Figure 5)

Lychnocanium grande rugosum Riedel, 1952, p. 6, pl. 1, fig. 1; Hays, 1965 , p. 175 , pl. 3, fig. 5

Abundance: Rare to common.

Occurrence: Pliocene.

Lychnocanoma sphaerothorax Weaver n. sp. (Plate 5, Figures 4, 5)

Description: Cephalis hemispherical, bearing a conical, apical horn, of approximately the same length, and a few small scattered 
pores. Separated from the thorax by a sharp collar stricture. Thorax truncate spherical with hexagonally framed subcircular to circular pores arranged in descussate rows. Three short, stout bladed feet, slightly diverted outward.

Measurements: Based on 25 specimens from Samples 323-3, CC and 323-5, CC: length of apical horn, 30-36 $\mu \mathrm{m}$; cephalis, $28-32 \mu \mathrm{m}$; thorax, $90-102 \mu \mathrm{m}$; feet, $30-48 \mu \mathrm{m}$; maximum diameter of the thorax, 96-120 $\mu \mathrm{m}$; diameter of thoracic pores, 4-12 $\mu \mathrm{m}$.

Abundance: Common.

Occurrence: Middle Miocene, Southern Ocean.

Range: Undetermined.

Type locality: DSDP Site 323, Core 3.

Repository: Holotype deposited at the U.S. National Museum, Washington D.C.

\section{Genus PERIPYRAMIS Haeckel}

Peripyramis Haeckel, 1881 , p. 428.

\section{Peripyramis circumtexta Haeckel} (Plate 1, Figure 5)

Peripyramis circumtexta Haeckel, 1887, p. 54, fig. 5; Riedel, 1958, p. 231 , pl. 2, fig. 8, 9; Petrushevskaya, 1968, p. 111-112, fig. 64, 1, 11.

Abundance: Rare to common.

Occurrence: Pliocene and Pleistocene.

\section{Genus SETHOCONUS Haeckel}

Sethoconus Haeckel, 1887, p. 1399.

\section{Sethoconus sp. Chen \\ (Plate 7, Figure 5)}

Sethoconus sp. Chen, 1975, p. 485, pl. 10, fig. 5, 6. Abundanco: Rare.

Occurrence: Middle Miocene.

\section{Sethoconus (?) dogeili Petrushevskaya (Plate 1, Figure 8)}

Sethoconus (?) dogeili Petrushevskaya, 1968, p. 94-95, fig. 53, 1, 11. Abundance: Rare.

Occurrence: Pleistocene.

\section{Genus STICHOCORYS Haeckel}

Stichocorys Haeckel, 1881, p. 438; Riedel and Sanfilippo, 1970, p. 530 .

\section{Stichocorys peregrina (?) (Riedel)}

Eucyrtidium elongatum peregrinum, Riedel, 1953, p. 812, pl. 85, fig. 2. Stichocorys peregrina (Riedel), Riedel and Sanfilippo, 1970, p. 530; Sanfilippo et al., 1973, p. 224, pl. 6, fig. 4.

Abundance: Rare.

Occurrence: Latest Miocene.

\section{Genus THEOCALYPTRA Haeckel}

Theocalyptra Haeckel, 1881, p. 434.

\section{Theocalyptra bicornis Popofsky}

Pterocorys bicornis Popofsky, 1908, p. 228-229, pl. XXXIV, fig. 7, 8. Theocalyptra bicornis (Popofsky), Riedel, 1958, p. 240, pl. 4, fig. 4; Chen, 1975, p. 491, pl. 13, fig. 1, 2 .

Theocalyptra (?) bicornis (Popofsky), Petrushevskaya, 1968, p. 124 127, fig. 71, II-IX; 72, I-IV.

Abundance: Rare.

Occurrence: Miocene through Pleistocene.

Theocalyptra bicornis spongothroax Chen

(Plate 2, Figures 1-4; Plate 6, Figures 2-4)

Theocalyptra bicornis spongothorax Chen, 1975, p. 489, pl. 12, fig. 1-3. Abundance: Rare.

Occurrence: Earliest Pliocene.

Genus THEOCORYS Haeckel

Theocorys Haeckel, 1881, p. 434; Riedel and Sanfilippo, 1970, p. 530.
Theocorys redondoensis (Campbell and Clark) (Plate 2, Figure 11)

Theocyrtis redondoensis Campbell and Clark, 1944, p. 49, pl. 7, fig. 4; Casey et al., 1972, pl. 2, fig. 3.

Theocorys redondoensis (Campbell and Clark), Kling, 1973, p. 638, pl. 11, fig. 26-28; Chen, 1975, p. 505, pl. 20, fig. $2,3$.

Abundance: Rare.

Occurrence: Pliocene.

\section{Family CANNOBOTRYIDAE Haeckel}

Cannobotryidae Haeckel, 1881, emend. Riedel, 1967b

\section{Genus SACCOSPYRIS Haecker}

Saccospyris Haecker, 1907, emend. Petrushevskaya, 1965

\section{Saccospyris conithorax Petrushevskaya}

Saccospyris conithorax Petrushevskaya, 1965, p. 98-99, fig. 11, Petrushevskaya, 1968, p. 150, fig. 85, 1 .

Abundance: Rare.

Occurrence: Pliocene and Pleistocene.

\section{Saccospyris antarctica Haecker \\ (Plate 1, Figure 6)}

Saccospyris antarctica Haecker, 1907, p. 124, fig. 10a, b; Haecker, 1908 , p. 447-448, pl. 84, fig. 584, 589, 590; Petrushevskaya, 1965 , p. 96-98, fig. 10; Petrushevskaya, 1968, p. 149-150, fig. 85, 11.

Botryopyle antarctica (Haecker), Riedel, 1958, p. 224-226, textfig. 13, pl. 4, fig. 12 (in part).

Abundance: Rare to common.

Occurrence: Pliocene and Pleistocene.

\section{ACKNOWLEDGMENTS}

I would like to express my appreciation to the Department of Geology at Florida State University for use of their scanning electron microscopy facility.

I wish to thank Dr. M.G. Dinkelman for review of the manuscript; D.S. Cassidy, Marianne Trinchitella, Yang Ja Chung, and Rosemarie Raymond, for technical assistance in preparing the manuscript.

I am also grateful to Percy Chen of Lamont-Doherty Geological Observatory for providing me with important unpublished radiolarian data from DSDP Leg 28, and the Deep Sea Drilling Project for allowing me to participate in the program on Leg 35.

Partial support for this investigation was provided by a Penrose grant from the Geological Society of America.

\section{REFERENCES}

Bailey, J.W., 1856. Notice of microscopic forms found in the soundings of the Sea of Kamtschatka - with a plate: Am. J. Sci., v. 22 , p. 1-6.

Bandy, O.L., Casey, R.E., and Wright, R.C., 1971. Late Neogene planktonic zonation, magnetic reversals and radiometric dates, Antarctic to the Tropics: Antarctic Res. Ser., Biol. Antarctic Seas, IV: Washington (Am. Geophys. Union), v. 15, p. 1-26.

Berggren, W.A., 1973. The Pliocene time-scale: calibration of planktonic foraminiferal and calcareous nannoplankton zones: Nature, v. 243, p. 391-397.

Bütschli, O., 1881. Beiträge zur Kenntnis der Radiolarienskelette, isobesondere der der Cyrtida: Z. Wiss. Zool., v. 36 , p. 485 .

1882. Radiolaria. In Bronn, H.G. (Ed.), Klassen und Ordnungen des Thier-Reichs, v. 1, p. 332.

Campbell, A.S. and Clark, B.L., 1944. Miocene radiolarian faunas from Southern California: Geol. Soc. Am., Spec. Paper 51, p. 1-76.

Casey, R.E., Price, A.B., and Swift, C.A., 1972. Radiolarian defintion and paleoecology of the late Miocene to early 
Pliocene in southern California. In Stinemeyer, E. H. (Ed.), The Pacific Coast Miocene biostratigraphic symposium, March 9-10, 1972: Bakersfield, California (Pacific Sec., Soc. Econ. Paleontol. Mineral.), p. 226.

Chen, P., 1975. Antarctic Radiolaria, Leg 28, Deep Sea Drilling Project. In Frakes, L.A., Hayes, D.E., et al., Initial Reports of the Deep Sea Drilling Project, Volume 28: Washington (U.S. Government Printing Office), p. $437-$ 513.

Ciesielski, P.F., 1975. Neogene and Oligocene silicoflagellates from cores recovered during Antarctic Leg 28, Deep Sea Drilling Project: Biostratigraphy and paleoecology. In Frakes, L.A., Hayes, D.E., et al., Initial Reports of the Deep Sea Drilling Project, Volume 28: Washington (U.S. Government Printing Office), p. 625-692.

Ciesielski, P.F. and Weaver, F.M., 1974. Early Pliocene temperature changes in the Antarctic Seas: Geology, v. 2, p. 511.

Cleve, P.T., 1899. Plankton collected by Swedish Expedition to Spitzbergen in 1898: $\mathrm{Kgl}$. Svensk. Vetensk, Akad. Handl., v. 32, p. 3.

Dreyer, F., 1889. Die Pylombildungen in vergleichendanatomischer und antwicklungsgeschichllicher Beziehung bei Radiolarien und bei Protisten uberhaupt, nebst System und Beschreibung neuer und der bis jetzt bekannten pylomatischen Spumellarian: Jen. Zeitschr., v. 23, p. 1.

Ehrenberg, C.G., 1838. Uber die bildung der kreidefelsen und des kreidemergels durch unsichtbare organismen: Abh. Kgl. Akad. Wiss. Berlin, p. 59.

1844. Uber 2 neue Lager von Gebirgsmassen aus Infusorien als Meeres-Absatz in Nord-Amerika und eine Vergleichung derselben mit den organischen KreideGebilden in Europa und Afrika: Kgl. Preuss. Akad. Wiss. Berlin, p. 57.

1847a. Uber eine halibiolithische, von Herrn R. Schomburgk entdeckte, vorherrschend aus mikroskopishen Polycystinen gebildete, Gebirgsmasse von Barbados: Kgl. Preuss. Akad. Wiss. Berlin, p. 382.

1847b. Uber die mikroskopischen kieselschaligen Polycystinen als mächtige Gebirgsmasse von Barbados und über das Verhältniss der aus mehr als 300 Neuen Arten bestehended ganz eigenthümlichen formengruppe jener Felsmasse zu den jetzt lebenden Thieren und zur Kreidebildung. Eine neue Anregung zur Erforschung des Erdlebens: Kgl. Preuss. Akad. Wiss. Berlin, p. 40.

1854. Die systematische characteristik der neuen mikroskopischen organismen des tiefen Atlantischen Oceans: Kgl. Preuss. Akad. Wiss. Berlin, p. 236.

1858. Kurze characteristik der 9 neuen genera und der 105 neuen species des ägäischen meeres und des tiefgrundes des Mittel-Meeres: Monats ber. Kgl. Preuss. Akad. Wiss. Berlin, v. 10.

1860. Ueber den Tiefgrund des Stillen Oceans zwischen Californien und des Sandwich-Inseln: Monatsber. Kgl. Preuss. Akad. Wiss. Berlin, p. 819.

1862. Die Tiefgrund-Verhältnisse des ozeans am Eingange der Davisstrasse und bei Island: Monatsber. Kgl. Preuss. Akad. Wiss. Berlin, p. 275.

1873. Grössere Felsproben des PolycystinenMergels von Barbados mit weiteren Erlauterungen: Monatsber. Kgl. Preuss. Akad. Wiss. Berlin, p. 213. 1875. Fortsetzung der mikrogeologischen Studien als Gesammt-Uebersicht der mikroskopischen Pälaontologie gleichartia analysirter Gebirgsarten der Erde, mit specieller Rücksicht auf den Plycystinen-Mergel von Barbados: Abh. Kgl. Akad. Wiss. Berlin, p. 1.

1887. Report on the Radiolaria collected by H.M.S. Challenger during the years 1873-76. Rept. Voy. Challenger, Zool., v. 18, p. 1-1803.
Friend, J.K. and Riedel, W.R., 1967. Cenozoic orosphaerid radiolarians from tropical Pacific sediments: Micropaleontology, v. 13, p. 217.

Goll, R.M., 1968. Classification and phylogeny of Cenozoic Trissocyclidae (Radiolaria) in the Pacific and Caribbean basings. Part I: J. Paleontol., v. 42 (6), p. 1409.

Haeckel, E., 1860. Abbildungen und diagnosen neuer gattungen und arten von lebenden Radiolarien des Mittelmeeres: Monatsber. Kgl. Preuss. Akad. Wiss. Berlin, p. 835.

1862. Die Radiolarien. (Rhizopoda Radiaria): Berlin (Reimer), 572 p; Atlas.

1881. Entwurf eines Radiolarien-Systems auf Grund von Studien der Challenger-Radiolarien: Jena. Z. Med. Naturwiss, v. 15, p. 418.

1887. Report on the Radiolaria collected by H.M.S. Challenger during the years 1873-76: Rept. Voy. Challenger, Zool., v. 18, p. 1-1803.

Haecker, V., 1907. Altertümliche Sphärellarien und Cyrtellarien aus grossen Meerestiefen: Arch. Protistenk., v. 10 , p. 114

1908. Tiefsee-Radiolarien: Wiss. Drgebn. dt. TiefsExped: Valdivia, v. 14, p. 1-476.

Hays, J.D., 1965. Radiolaria and Late Tertiary and Quaternary history of Antarctic seas: Antarctic Res. Ser., Biol. Antarctic Seas, II: Washington (Am. Geophys. Union), v. 5 , p. $125-184$.

1970. Stratigraphy and evolutionary trends of Radiolaria in North Pacific deep-sea sediments. In Hays, J.D., Geological investigations of the North Pacific: Geol. Soc. Am. Mem. 126, p. 185.

Hays, J.D. and Opdyke, N.D., 1967. Antarctic Radiolaria, magnetic reversals, and climatic change: Science, v. 158 , p. 1001

Jörgensen, E., 1905. The protist plankton and the diatoms in bottom samples: Bergens Mus. Skr., p. 49-151, 195-225.

Keany, J. and Kennett, J.P., 1972. Pliocene-early Pleistocene paleoclimatic history recorded in Antarctic-Subantarctic deep-sea cores: Deep-Sea Res., v. 19, p. 529.

Kennett, J.P. and Brunner, C.A., 1973. Antarctic Late Cenozoic glaciation: Evidence for initiation of ice-rafting and inferred bottom water activity: Geol. Soc. Am. Bull., v. 84 , p. 2043

Kennett, J.P. and Watkins, N.D., 1974. Late Miocene-early Pliocene paleomagnetic stratigraphy, paleoclimatology, and biostratigraphy in New Zealand: Geol. Soc. Am. Bull., v. 85 , p. 1385.

Kling, S.A., 1973. Radiolaria from the eastern North Pacific, Deep Sea Drilling Project, Leg 18. In Kulm, L.D., von Huene, R., et al., Initial Reports of the Deep Sea Drilling Project, Volume 18: Washington (U.S. Government Printing Office), p. 617-671.

Ling, H.Y., Stadium, C.J., and Welch, M.L., 1971. Bering Sea surface sediments. In A. Farinacii (Ed.), Proc. 2nd Plankt. Conf. Roma 1970: Rome (Tecnoscienza), v. 2, p. 705 .

Martin, G.C., 1904. Radiolaria: Baltimore (Maryland Geol. Surv., Gen Ser.), p. 447-459.

McCollum, D.W., 1975. Antarctic Cenozoic diatoms: Leg 28, Deep Sea Drilling. In Frakes, L.A., Hayes, D.E., et al., Initial Reports of the Deep Sea Drilling Project: Volume 28: Washington (U.S. Government Printing Office), p. 515-572.

Müller, J., 1858. Uber die Thalassicollen, Polycystinen und Acanthometren des Mittelmeeres: Kgl. Akad. Wiss. Berlin, Abh., Jahre 1858, p. 1.

Nakaseko, K., 1959. On superfamily Liosphaericae (Radiolaria) from sediments in the sea near Antarctica, 1, On Radiolaria from sediments in the sea near Antarctica: Seto 
Marine Biol. Lab., Spec. Publ., Biol. Results, Japanese Antarctic Exped., p. 2.

Nigrini, C., 1967. Radiolaria in pelagic sediments from the Indian and Atlantic oceans: Scripps Inst. Oceanogr. Bull. 11, $125 \mathrm{p}, 9 \mathrm{pl}$.

Opdyke, N.D., 1972. Paleomagnetism of deep-sea cores: Geophys. Space Phys. Rev., v. 10, p. 213.

Petrushevskaya, M.G., 1962. Znachenie rosta skeleta radiolyarii dlya ikh sistematiki: Zool. Zhr., v. XII, p. 331-341.

1964. O gomologiyakh elementov vnutrennego skeleta nekotorykĭ radiolyarii Nassellaria (Homologies of elements of the internal skeleton of Nassellarian radiolarians): Zool. Zh., v. 43 , p. 1121.

1965. Osobennosti konstruktsii skeleta radiolyarii Botryoidae (otr. Nassellaria): Tr. Zool. Inst., Leningrad, v. 35 , p. $79-118$.

1968. Gomologii v skeletakh radiolyarii Nassellaria. 2. Osnovnye skeletnye dugi slozhnoustroennykh tsefalisov Cyrtoidae i Botryoidae: Zool. Zh., v. 47, p. $1766-1776$.

1969. Gomologii v skeletakh radiolyarii Nassellaria. 3. Sagittalno koltso i perifericheskii skelet semeistv Stephoidae i Spyroidae: Zool Zh., v. 48, p. 642-657.

1975. Cenozoic radiolarians of the Antarctic, Leg 29, DSDP, In Kennett, J.P., Houtz, R.E., et al., Initial Reports of the Deep Sea Drilling Project, Volume 29: Washington (U.S. Government Printing Office), p. 541676.

Petrushevskaya, M. and Kozlova, G., 1972. Radiolaria: Leg 14, Deep Sea Drilling Project. In Hayes, D.E., Initial Reports of the Deep Sea Drilling Project, Volume 14: Washington (U.S. Government Printing Office), p. 459648

Popofsky, A., 1908. Die radiolarien der Antarktis: Deut. Sudpolar Exped. 1901-1903: v. 10, Zool., v. 3, p. 185.

1912. Die Sphaerellarien des warmwassergebietes:

Deut. Südpolar Exped. 1901-1903, v. 13, Zool., v. 5, p. 75 .

Riedel, W.R., 1952. Tertiary radiolaria in western Pacific sediments: Medd. Oceanogr. Inst. Göteborg, v. 19, p. 122 .

1953. Mesozoic and late Tertiary Radiolaria of Rotti: J. Paleontol., v. 27 , p. $805-813$.

1958. Radiolaria in Antarctic sediments: B.A.N.Z.

Antarctic Res. Exped. Rept., Ser. B, v. 6, p. 217.

1959. Oligocene and lower Miocene Radiolaria in

tropical Pacific sediments: Micropaleontology, v. 5, p. 285 .

1967a. Some new families of Radiolaria: Geol. Soc. London Proc., v. 1640, p. 148. 1967b. Subclass Radiolaria. In Harland, W.B., et al. (Eds.), The fossil record: London (Geol. Soc. London), p. 291.

, 1971. Systematic classification of polycystine Radiolaria. In Funnell, B.M. and Riedel, W. R. (Eds.), The micropaleontology of oceans: Cambridge (Cambridge University Press), p. 649.

Sanfilippo, A. and Riedel, W.R., 1970. Post-Eocene "closed" theoperid radiolarians: Micropaleontology, v. 16, p. 446

1973. Cenozoic Radiolarians. In Worzel, J.L., Bryant, W., et al., Initial Reports of the Deep Sea Drilling Project, Volume 10: Washington (U.S. Government Printing Office), p. 457.

Sanfilippo, A., Burckle, L.H., Martini, E., and Riedel, W.R., 1973. Radiolarians, diatoms, silicoflagellates and calcareous nannofossils in Mediterranean Neogene: Micropaleontology, v. 19, p. 209.

Vinassa de Regny, P.E., 1900. Radiolari Miocenici Italiani: Roy. Accad. Sci. Bologna Mem., v. 8, p. 565-595.

Watkins, N.D. and Kennett, J.P., 1972. Regional sedimentary disconformities and Upper Cenozoic changes in bottom water veolcities between Australasia and Antarctica: Antarctic Res. Ser., v. 19, p. 273.

Weaver, F.M., 1973. Pliocene paleoclimatic and paleoglacial history of East Antarctica recorded in deep-sea piston cores: Florida State Univ., Sedimentological Res. Lab. Contrib. 36, p. 1-142.

Weaver, F.M. and Ciesielski, P.F., 1973. Pliocene paleoclimatic history recorded in Antarctic deep-sea cores: Geol. Soc. Am., Abstract with Programs, v. 5, p. 856. 1974. Pliocene paleotemperatures and regional correlations, Southern Ocean: Antarctic J. U.S., v. 9, p. 251.

Zittel, K.A., 1876. Ueber einige fossile radilarien aus der norddeutschen Kreide: Z. Deut. Geol. Ges., v. 28, p. 7586

\section{PLATES}

Plates 6-9 are scanning electron micrographs taken on an International Scientific Instruments, Super Mini Scan. Some illustrations on plates 2, 6, 7, and 8 were prepared from Samples of ELTANIN piston Core 34-5.

Preparations of type specimens of Lychnocanoma sphaerothorax and Triceraspyris coronatus are deposited at the United States National Museum, Washington, D.C. 



\section{PLATE 1}

Figure 1 Actinomma yosii Nakaseko.

Sample 324-1-5, 58-59 cm; $\times 225$.

Figure 2 Triceraspyris antarctica (Haecker). Sample 324-1-4, 68-70 cm; $\times 240$.

Figure 3 Triceraspyris antarctica (Haecker). Sample 324-1-5, 58-59 cm; $\times 225$.

Figure 4 Saturanlis circularis Haeckel.

Sample 324-1-3, 82-84 cm; $\times 160$.

Figure $5 \quad$ Peripyramis circumtexta Haeckel.

Sample 324-1-2, 138-140 cm; $\times 192$.

Figure 6 Saccospyris antarctica Haecker.

Sample 324-1-1, 143-145 cm; $\times 280$.

Figure $7 \quad$ Cycladophora davisiana Ehrenberg. Sample 324-1-1, 143-145 cm; $\times 295$.

Figure $8 \quad$ Sethoconus (?) dogeili Petrushevskaya. Sample 324-1-3, 82-84 cm; $\times 225$.

Figure 9 Eucyrtidium calvertense Martin. Sample 322-1-1, 44-46 cm; $\times 260$.

Figure 10 Helotholus vema Hays.

Sample 322-1-3, 19-21 cm; $\times 320$.

Figure 11 Helotholus vema Hays.

Sample $322-1-3,19-21 \mathrm{~cm} ; \times 300$.

Figure 12 Desmospyris spongiosa Hays.

Sample 322-2-2, 97-99 cm; $\times 260$. 
PLATE 1

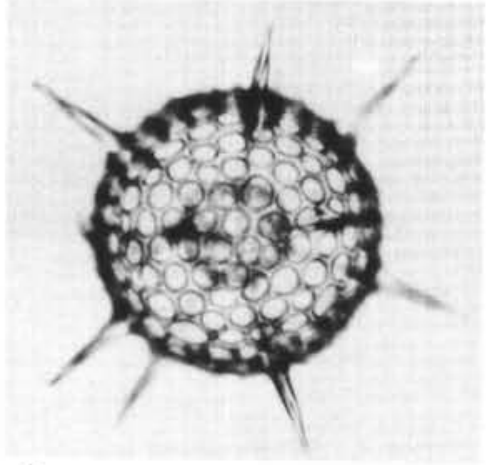

1

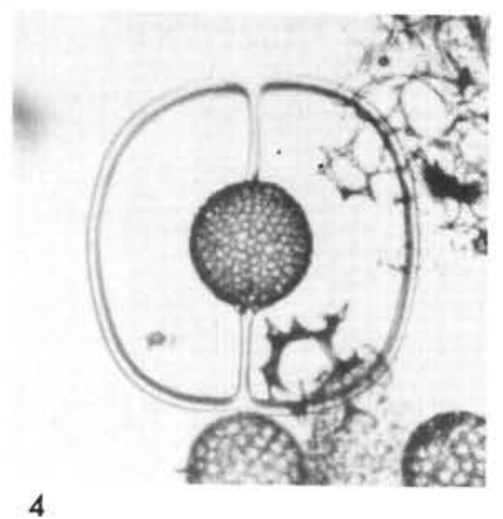

4

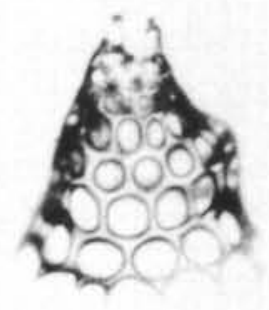

7

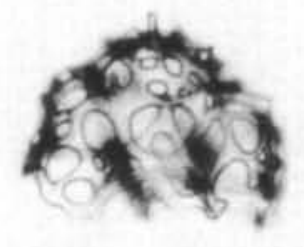

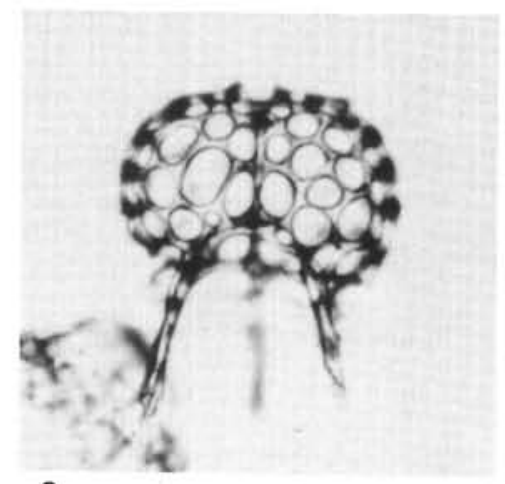

2
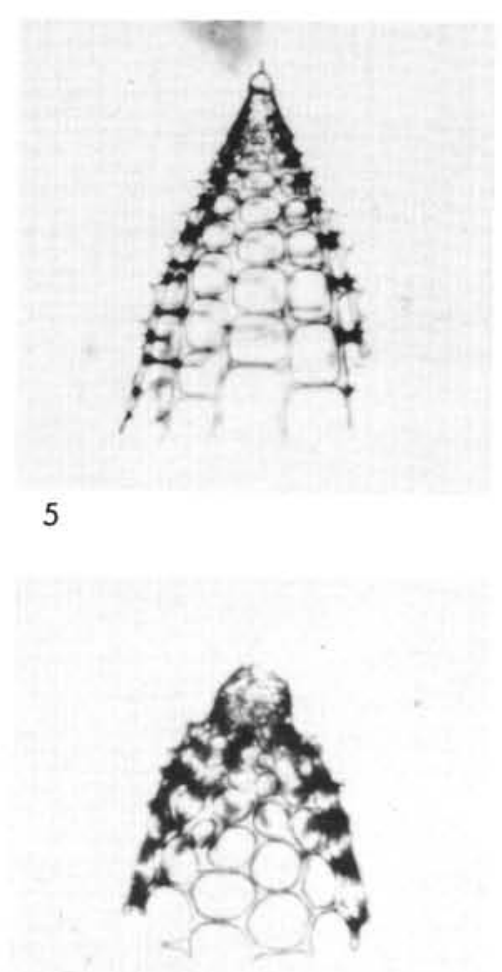

8

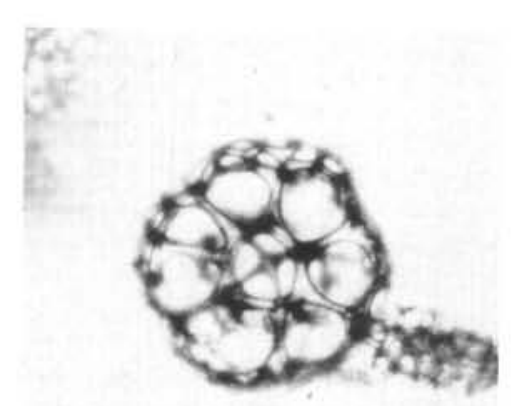

11

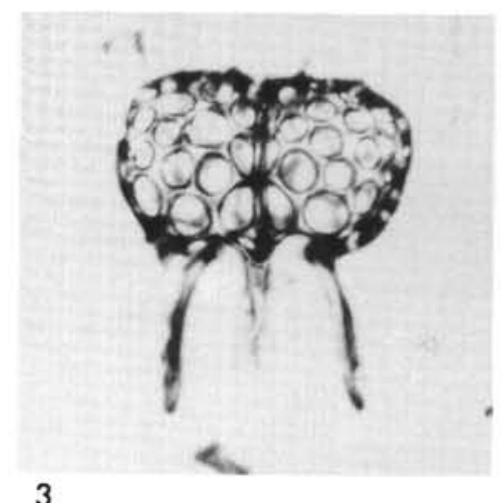

3

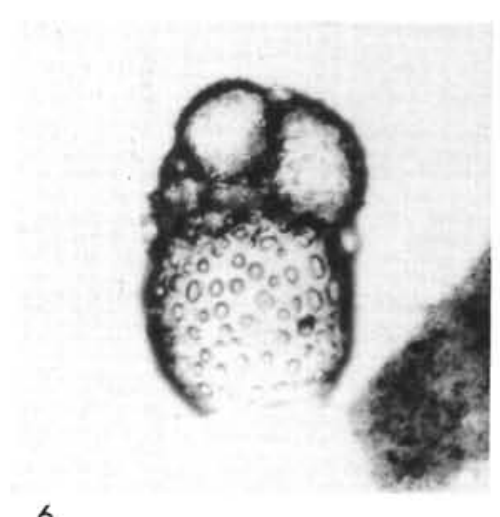

6
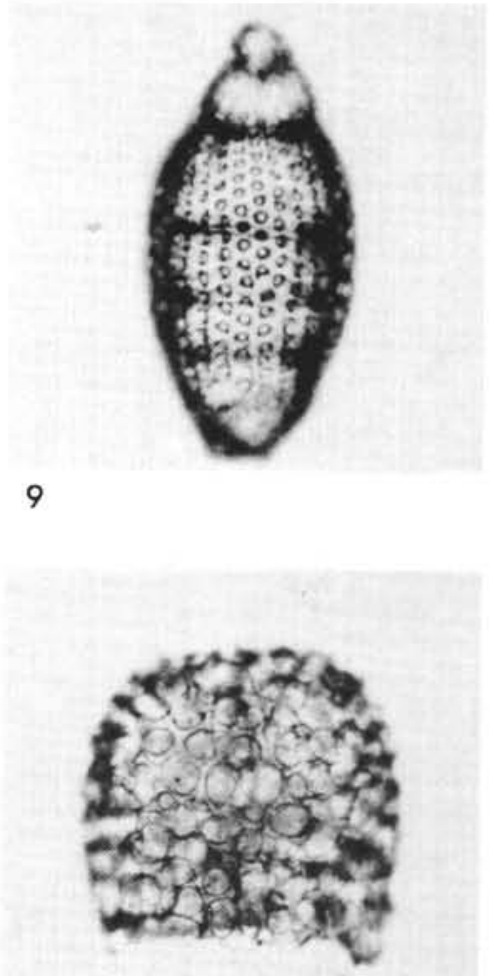


\section{PLATE 2}

Figure 1 Theocalyptra bicornis spongothorax Chen. ELTANIN $34-5,360 \mathrm{~cm} ; \times 430$.

Figure 2 Theocalyptra bicornis spongothorax Chen. ELTANIN 34-5; $360 \mathrm{~cm} ; \times 350$.

Figure 3 Theocalyptra bicornis spongothorax Chen. Sample 325-5, CC; $\times 380$.

Figure 4 Theocalyptra bicornis spongothorax Chen. Sample 325-5, CC; $\times 380$.

Figure $5 \quad$ Triceraspyris coronatus n. sp.

Sample 323-2-1, 132-134 cm; $\times 270$.

Figure 6 Triceraspyris coronatus n. sp.

Sample 323-2-1, 132-134 cm; $\times 245$.

Figure 7 Dendrospyris haysi Chen.

Sample 323-2-1, 132-134 cm; $\times 375$.

Figure 8 Dendrospyris haysi Chen.

Sample 322-2-2, 97-99 cm; $\times 360$.

Figure 9 Dendrospyris haysi Chen.

ELTANIN 34-5, $380 \mathrm{~cm} ; \times 320$.

Figure $10 \quad$ Lychnocanoma grande rugosum (Riedel). Sample 323-2-1, 132-134 cm; $\times 260$.

Figure 11 Theocorys redondoensis (Campbell and Clark). Sample 322-1-1, 44-46 cm; $\times 320$.

Figure 12 Eucyrtidium sp. aff. E. inflatum Kling. Sample 323-2, CC; $\times 250$. 
PLATE 2
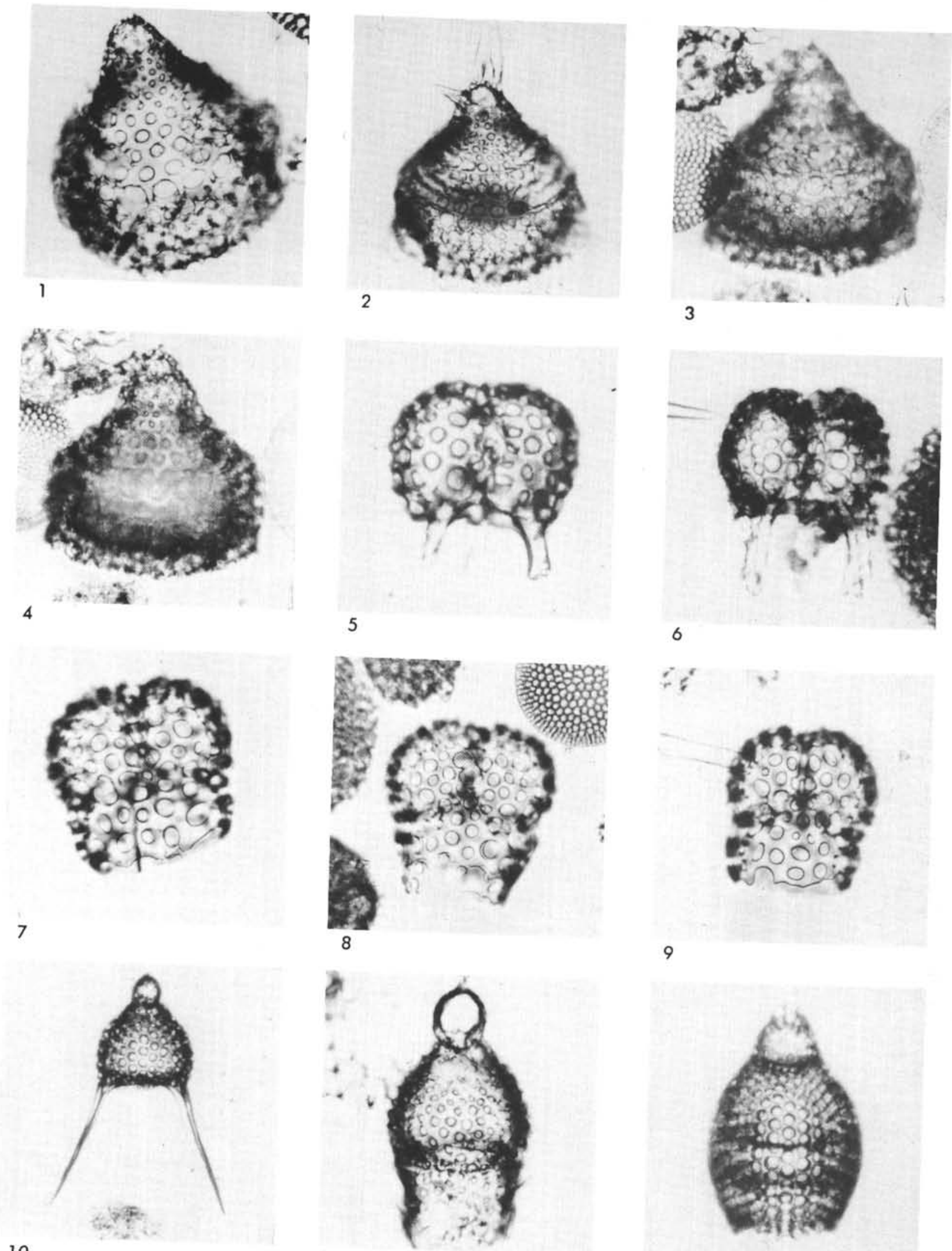

9

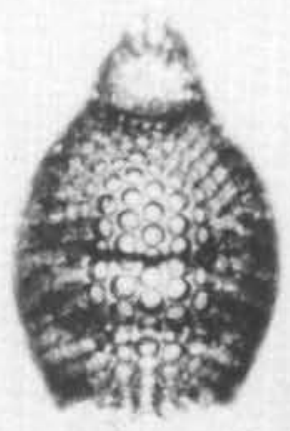




\section{PLATE 3}

Figure 1 Cyrtocapsella tetrapera Haeckel. Sample 323-3, CC; $\times 290$.

Figure $2 \quad$ Cyrtocapsella tetrapera (?) Haeckel. Sample 323-3, CC; $\times 260$.

Figure 3 Cyrtocapsella tetrapera Haeckel. Sample 323-3, CC; $\times 330$.

Figure $4 \quad$ Cyrtocapsella isopera Chen. Sample 323-6, CC; $\times 360$.

Figure $5 \quad$ Cyrtocapsella isopera Chen. Sample 323-6, CC; $\times 390$.

Figure $6 \quad$ Cyrtocapsella isopera Chen. Sample 323-6, CC; $\times 420$.

Figure $7 \quad$ Cyrtocapsella japonica (?) (Nakaseko). Sample 323-3, CC; $\times 340$.

Figure $8 \quad$ Cyrtocapsella cornuta (?) Haeckel. Sample 323-3, CC; $\times 230$.

Figure 9 Dendrospyris megalocephalis Chen. Sample 323-3, CC; $\times 260$.

Figure 10 Dendrospyris megalocephalis Chen. Sample 323-3, CC; $\times 310$.

Figure $11 \quad$ Oroscena sp. Sample 323-3, CC; $\times 90$ (spine). 
PLATE 3
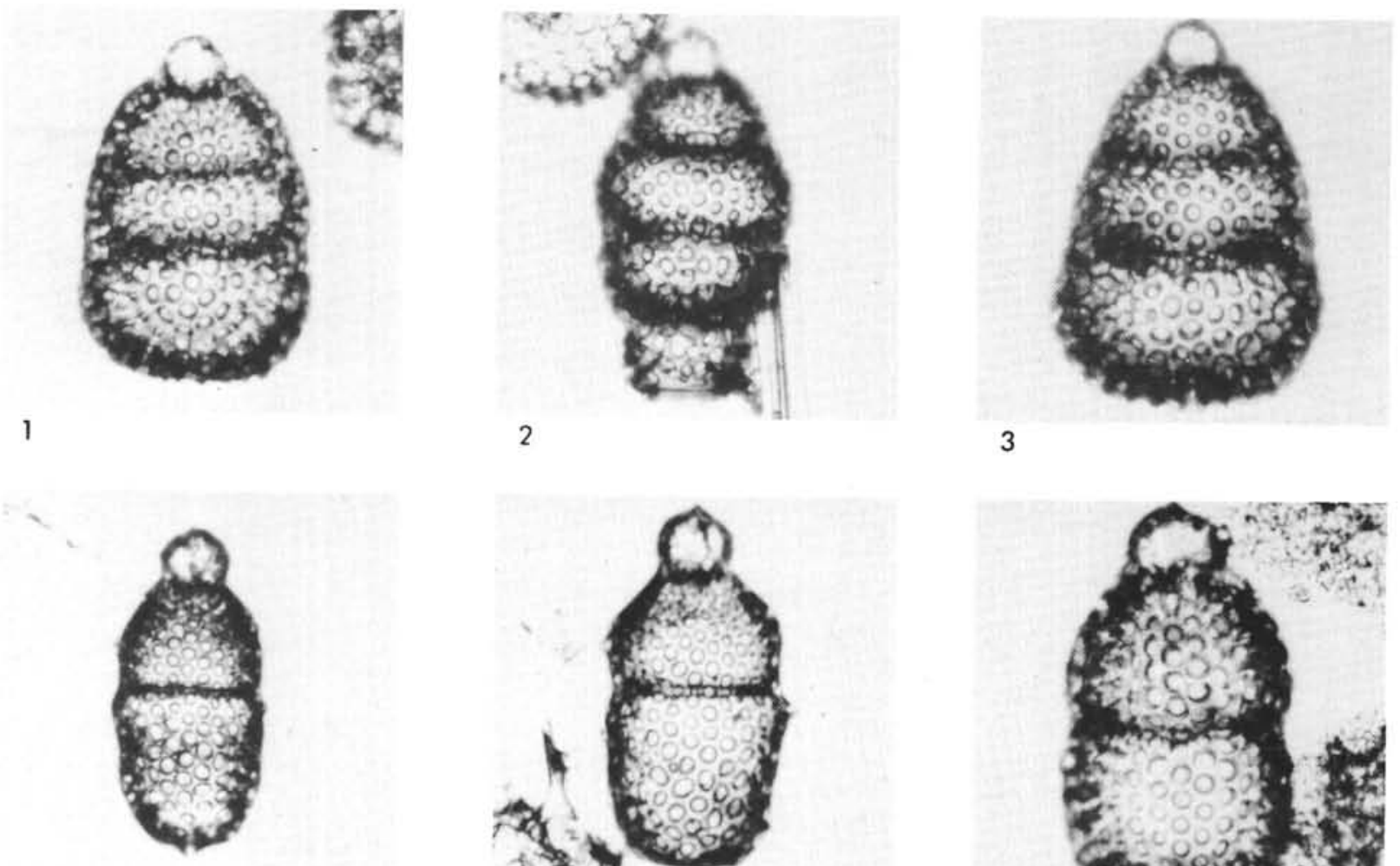

4
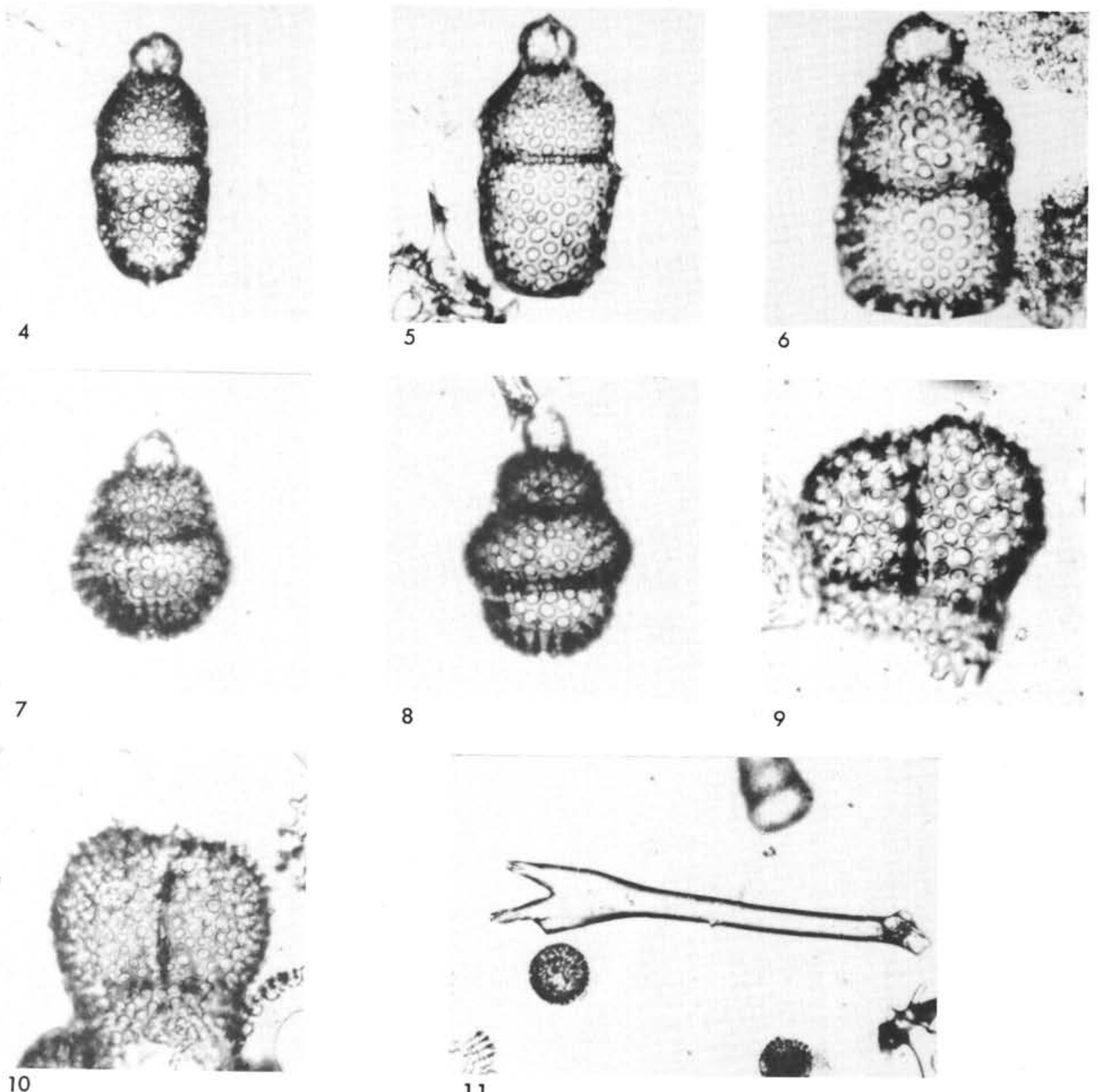

8

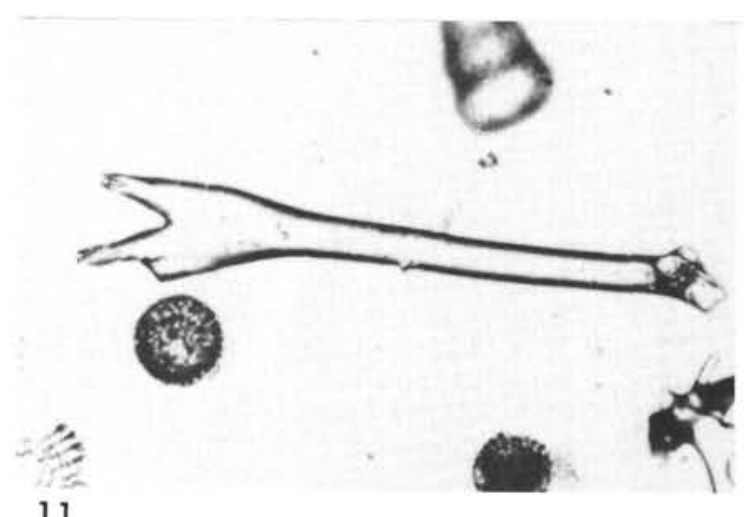




\section{PLATE 4}

Figure $1 \quad$ Eucrytidium punctatum (Ehrenberg) group. Sample 323-6, CC; $\times 230$.

Figure 2 Eucrytidium punctatum (Ehrenberg) group. Sample 323-6, CC; $\times 260$.

Figure 3 Eucrytidium cienkowskii Haeckel group. Sample 323-6, CC; $\times 230$.

Figure $4 \quad$ Eucrytidium cienkowskii Haeckel group. Sample 323-6, CC; $\times 230$.

Figure $5 \quad$ Eucyrtidium cienkowskii Haeckel group. Sample 323-6, CC; $\times 350$.

Figure $6 \quad$ Lophocyrtis regipileus Chen. Sample 323-7, CC; $\times 280$.

Figure $7 \quad$ Lophocyrtis regipileus (?) Chen. Sample 323-6, CC; $\times 245$.

Figure $8 \quad$ Lophocyrtis regipileus (?) Chen. Sample 323-6, CC; $\times 330$.

Figure 9 Lophocyrtis golli Chen. Sample 323-6, CC; $\times 310$.

Figure 10 Lophocyrtis golli Chen. Sample 323-6, CC; $\times 300$.

Figures 11, 12 Recrystallized Cretaceous radiolarians. Sample 323-18-3, 143-145 cm; $\times 150$. 

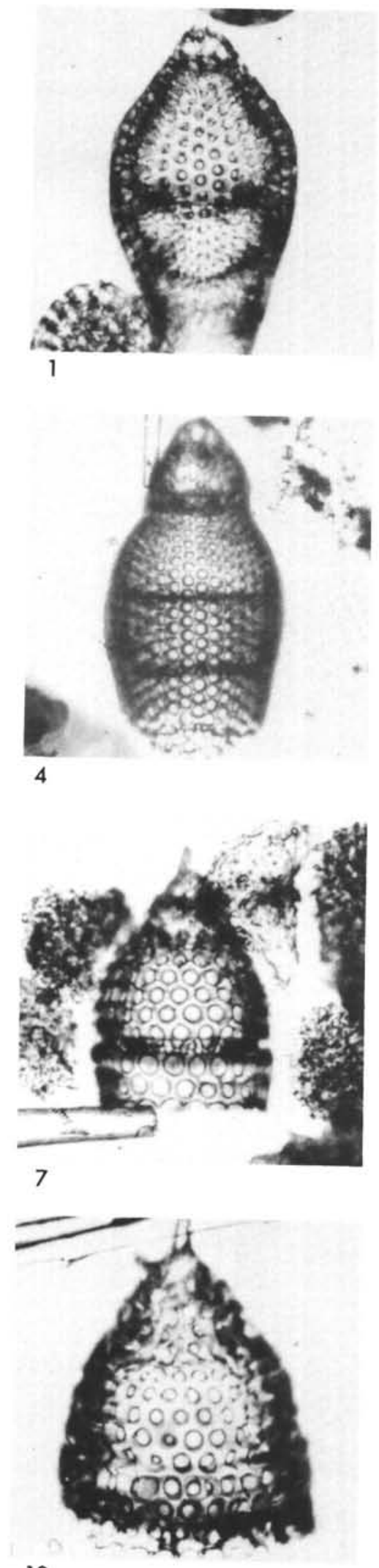

10
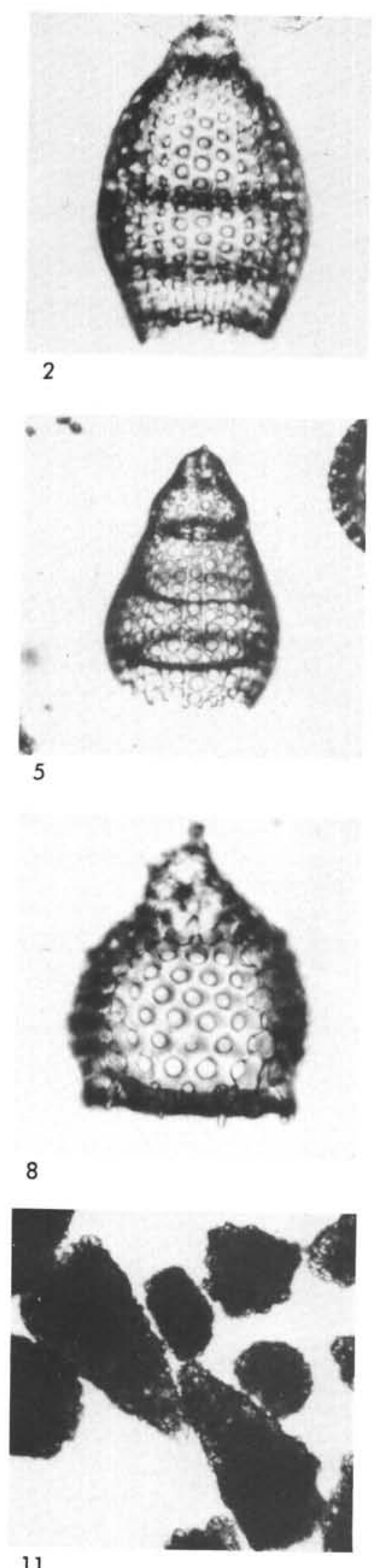
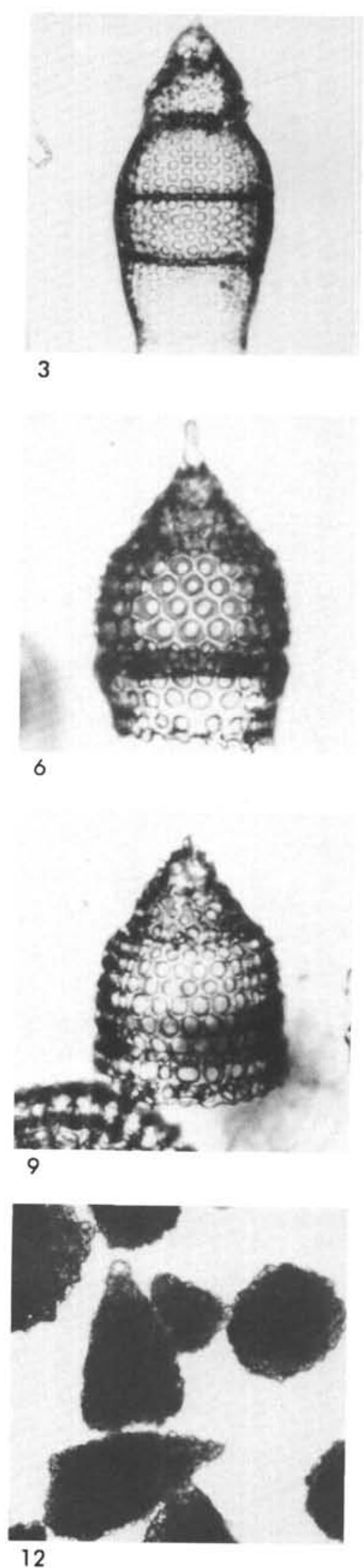


\section{PLATE 5}

Figure $1 \quad$ Druppatractus sp. Chen. Sample 323-2, CC; $\times 240$.

Figure 2 Druppatractus sp. Chen. Sample 323-2, CC; $\times 240$.

Figure 3 Stylatractus universus Hays. Sample 323-2, CC; $\times 280$ (early Pliocene form).

Figure $4 \quad$ Lychnocanoma sphaerothorax n. sp. Sample 323-3, CC; $\times 230$.

Figure $5 \quad$ Lychnocanoma sphaerothorax n. sp. Sample 323-3, CC; $\times 230$.

Figure 6 Stylatractus universus Hays. Sample 323-2, CC; $\times 280$ (early Pliocene form).

Figure $7 \quad$ Cannartus sp. aff. C. mammiferus (Haeckel). Sample $325-9-2,80-82 \mathrm{~cm} ; \times 260$ (partially recrystallized specimen).

Figure $8 \quad$ Cannartus sp. aff. C. mammiferus (Haeckel). Sample 325-9-2, 80-82 cm; $\times 260$ (arrows point to protuberences).

Figure 9 Cannartus sp. aff. C. prismaticus (Haeckel). Sample 325-10-1, 89-91 cm; $\times 250$. 
PLATE 5
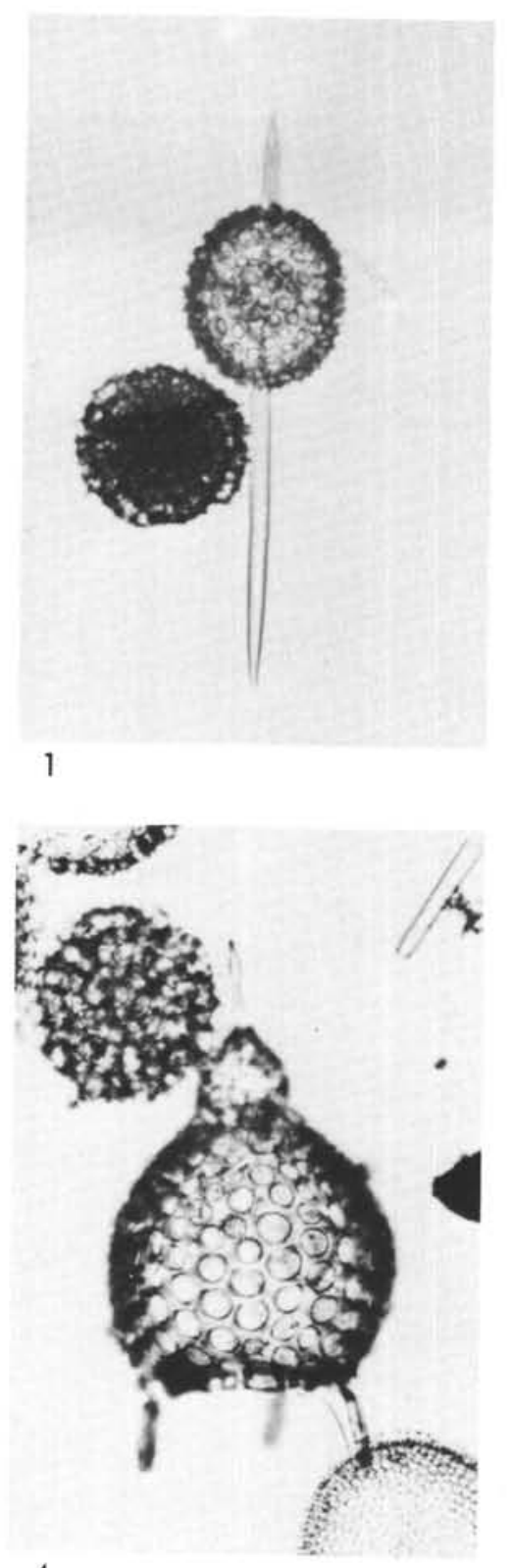

4

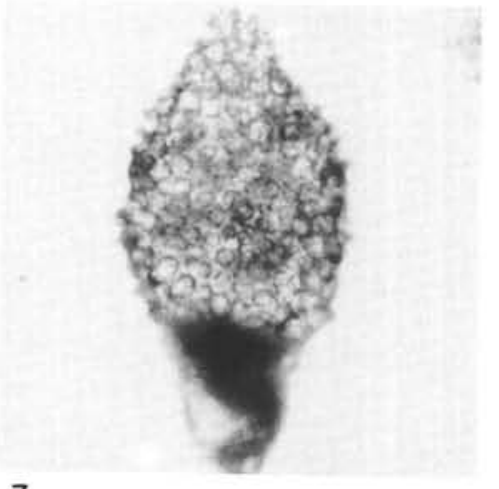

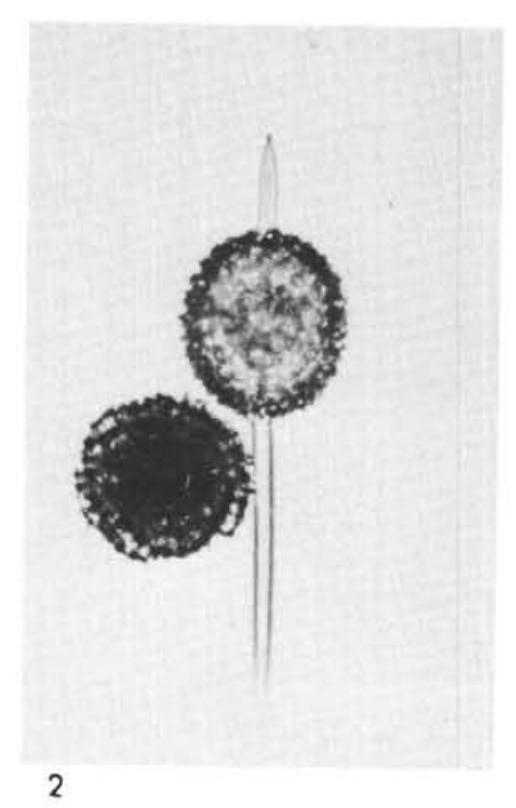
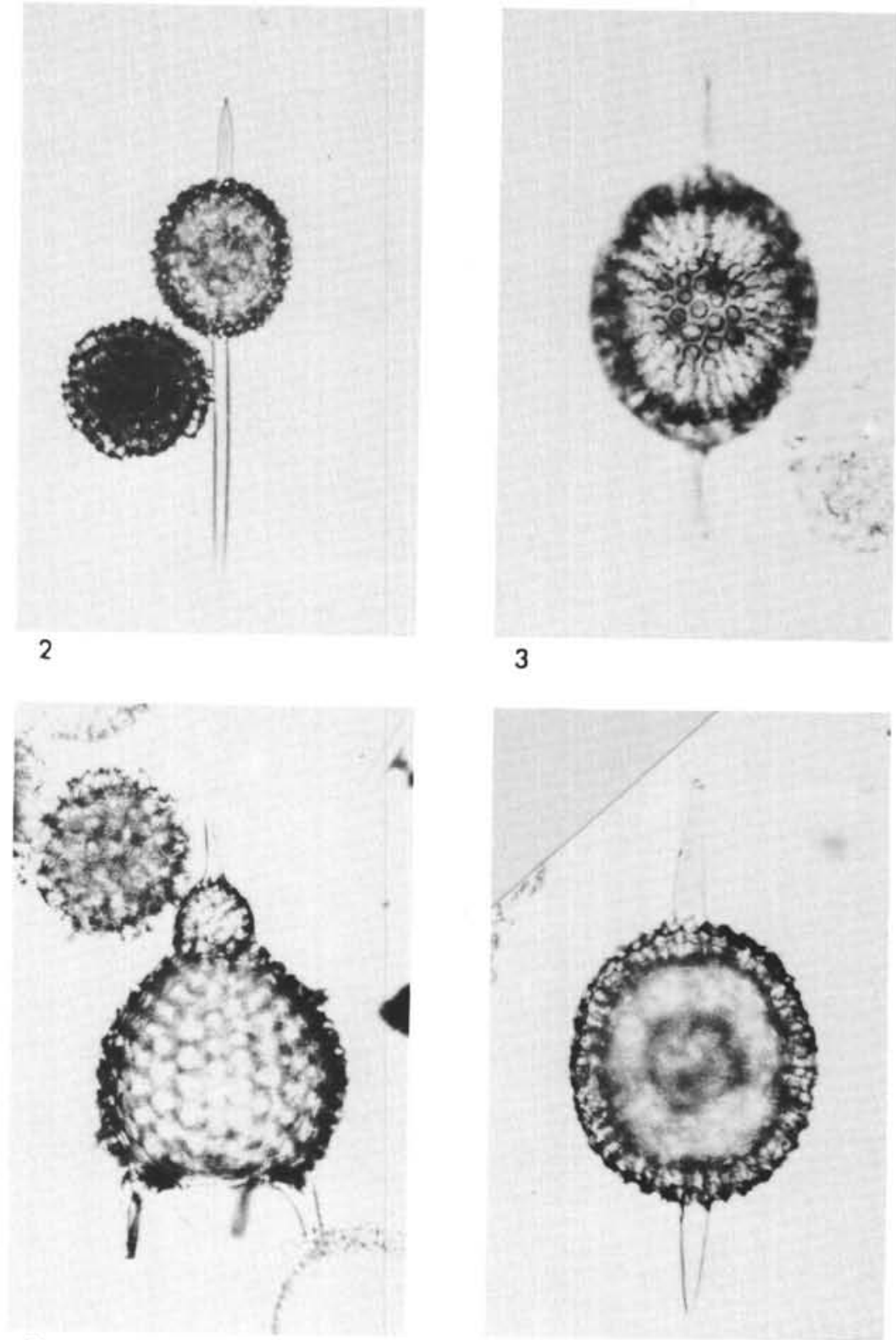

5
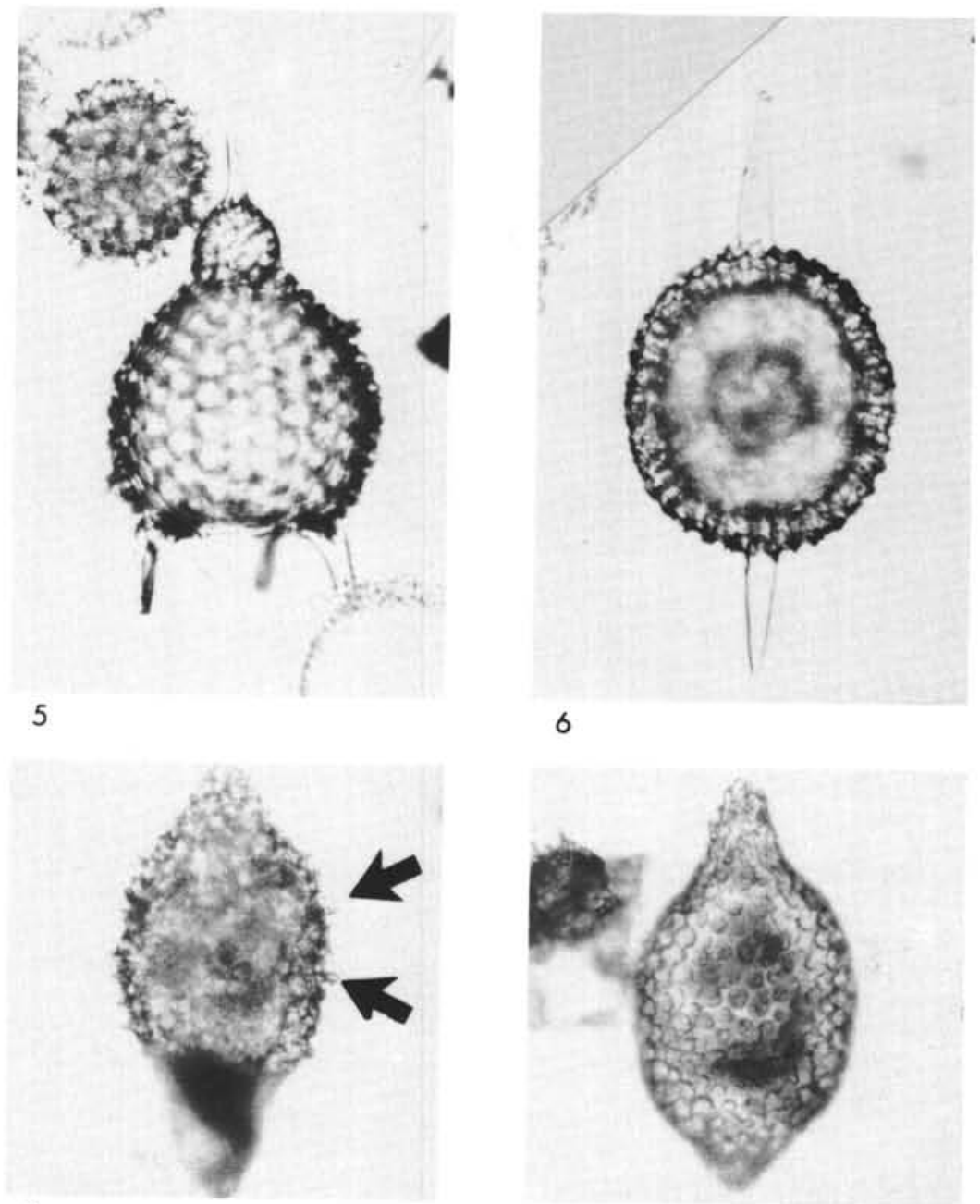

8

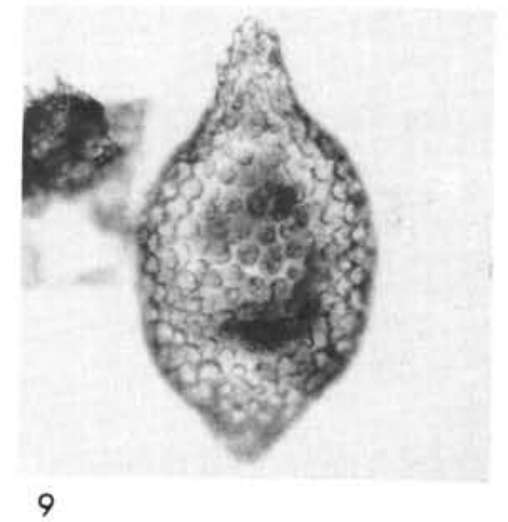




\section{PLATE 6}

Figure $1 \quad$ Druppatractus sp. Chen. Sample $323-2$, CC; $\times 270$.

Figure 2 Theocalyptra bicornis spongothorax Chen. Sample 325-5, CC; $\times 340$.

Figure 3 Theocalyptra bicornis spongothorax Chen. Sample 325-5, CC; $\times 310$.

Figure $4 \quad$ Theocalyptra bicornis spongothorax Chen. ELTANIN 34-5, $360 \mathrm{~cm}$; $\times 350$.

Figure $5 \quad$ Stylatractus universus Hays. Sample 323-2, CC; $\times 310$. (Early Pliocene form, specimen tilted along polar axis.)

Figure 6 Stylatractus universus Hays. Sample 323-2, CC; $\times 340$ (early Pliocene form).

Figure $7 \quad$ Stylatractus universus Hays. Sample 324-1-5, 58-59 cm; $\times 280$ (Pleistocene form).

Figure $8 \quad$ Triceraspyris coronatus n. sp.

Sample 323-2-1, 132-134 cm; $\times 320$.

Figure 9 Triceraspyris coronatus n. sp.

Sample 323-2-1, 132-134 cm; $\times 320$. 

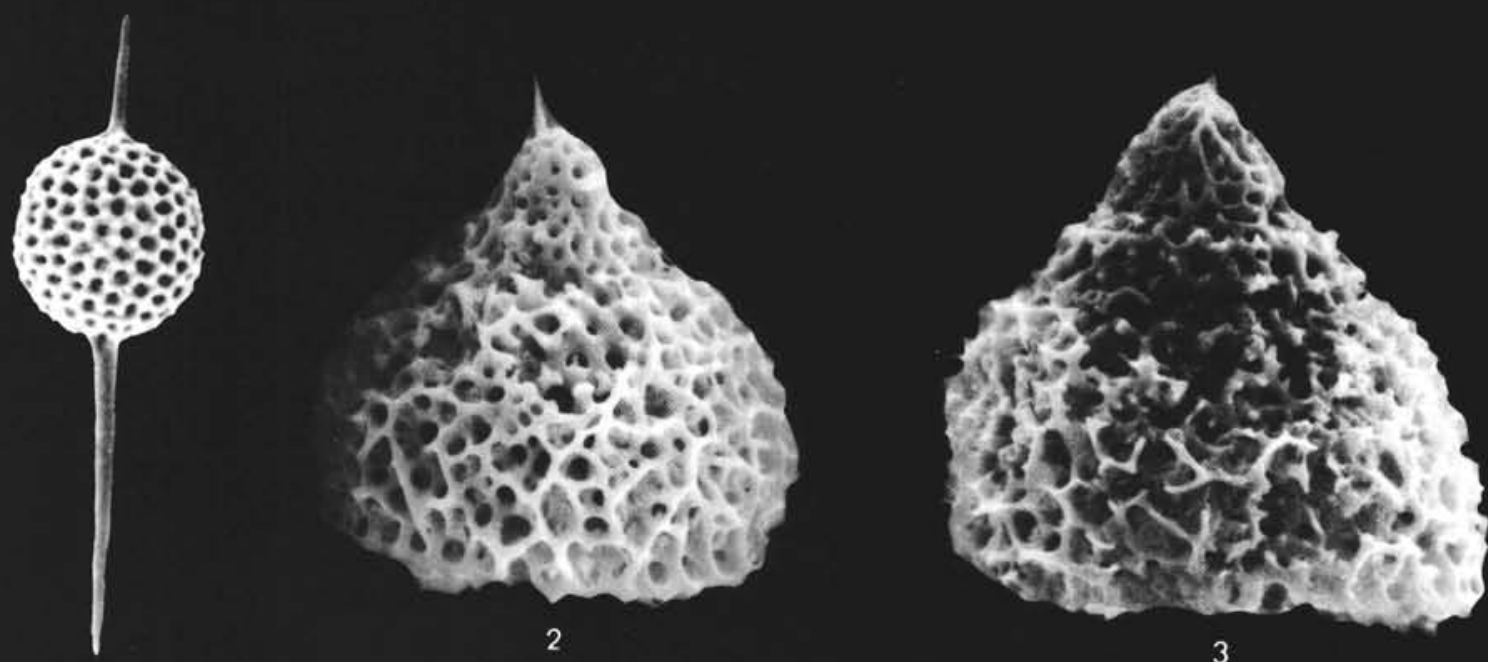

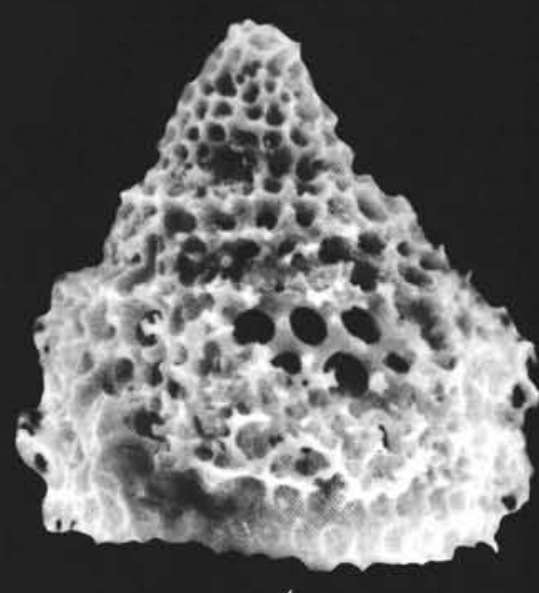

4

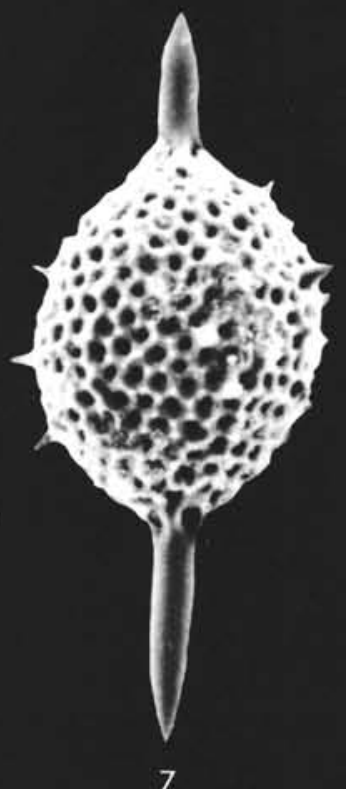

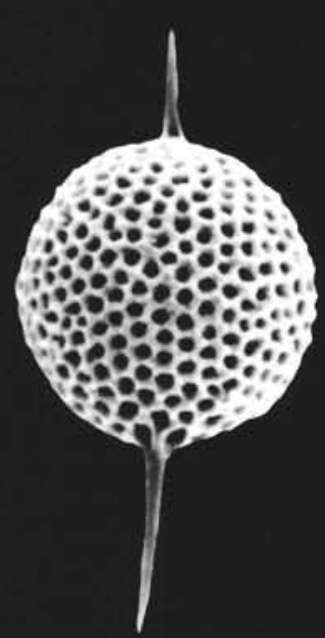

5

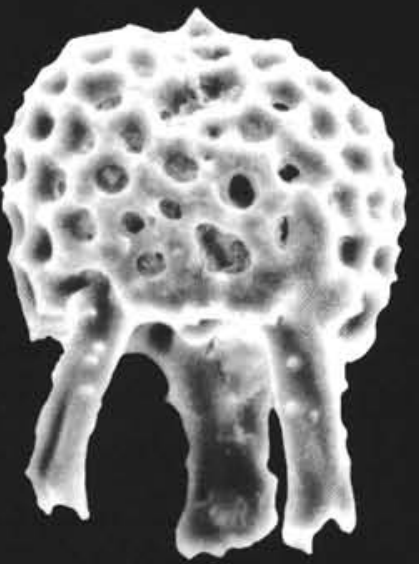

8

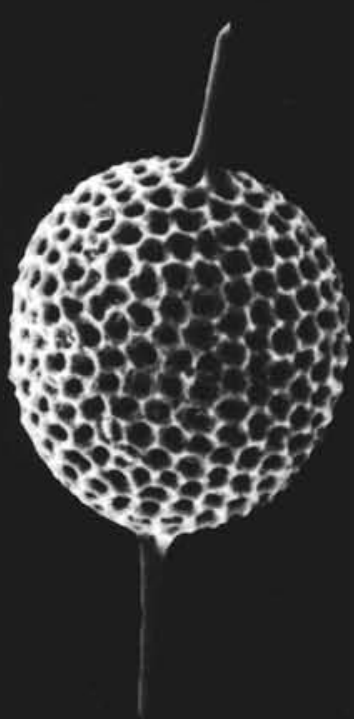

6

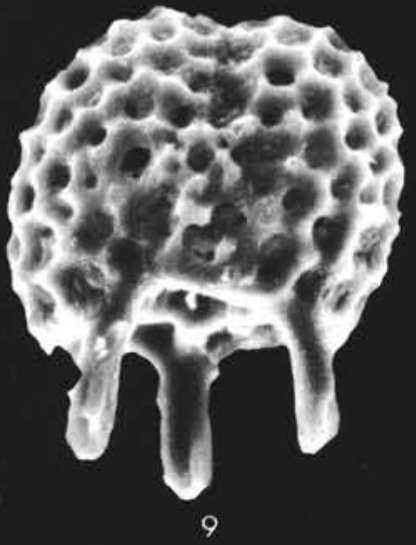




\section{PLATE 7}

Figure 1

Figure 2

Figure 3

Figure 4

Figure 5

Figure 6

Figure 7

Figure 8

Figure 9
Prunopyle hayesi Chen. ELTANIN 34-5, $440 \mathrm{~cm} ; \times 320$.

Prunopyle hayesi Chen. ELTANIN 34-5, $440 \mathrm{~cm} ; \times 160$.

Prunopyle hayesi Chen. ELTANIN 34-5, $440 \mathrm{~cm} ; \times 190$.

Dendrospyris haysi Chen. Sample 323-2-1, 132-134 cm; $\times 340$.

Sethoconus sp. Chen.

Sample 323-3, CC; $\times 360$.

Prunopyle titan (?) Campbell and Clark. Sample 323-1, CC; $\times 130$.

Lophocyrtis regipileus Chen. Sample 323-6, CC; $\times 360$.

Lophocyrtis regipileus Chen. Sample 323-6, CC; $\times 390$.

Lophocyrtis regipileus (?) Chen. Sample 323-6, CC; $\times 340$. 

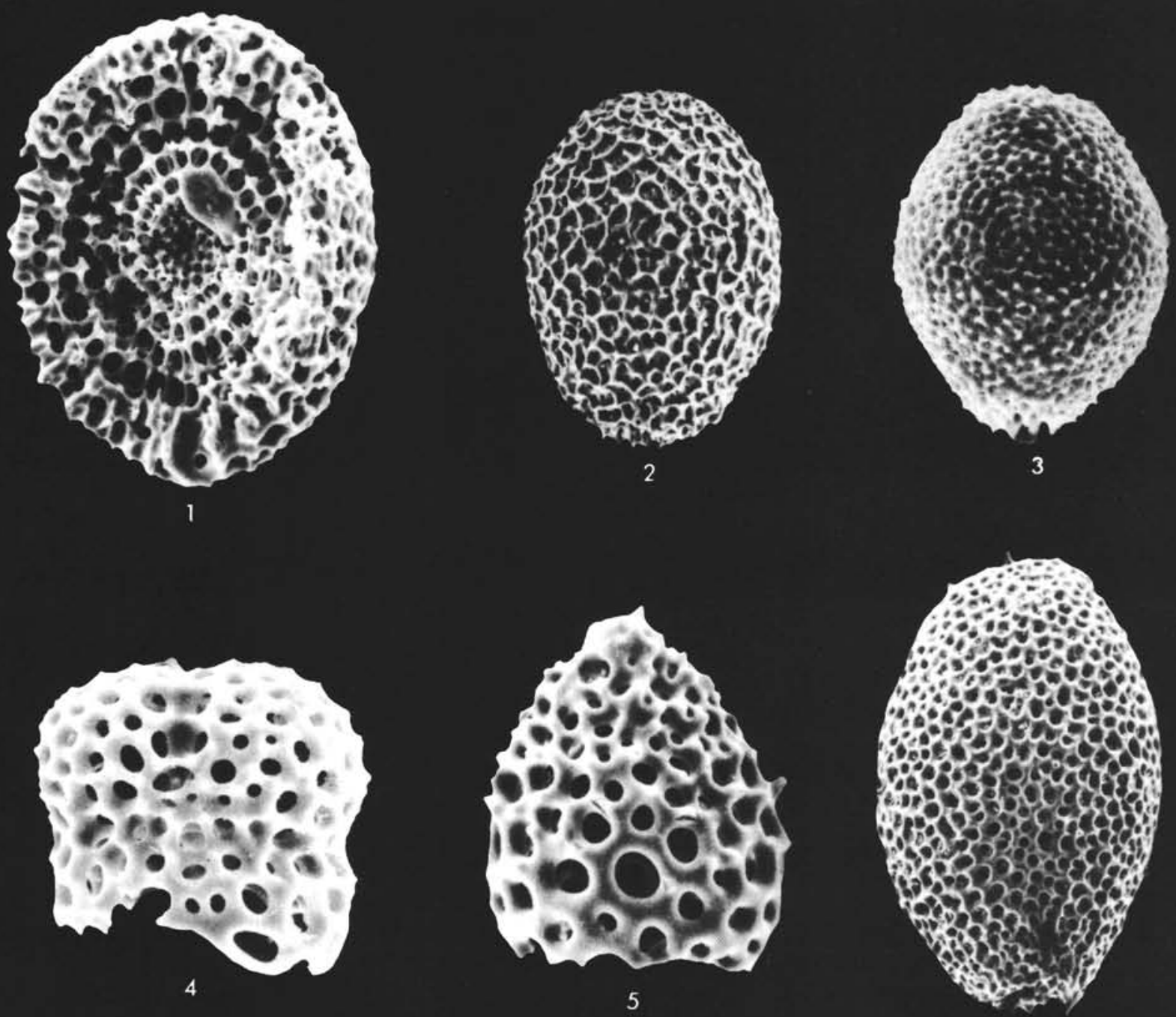

6

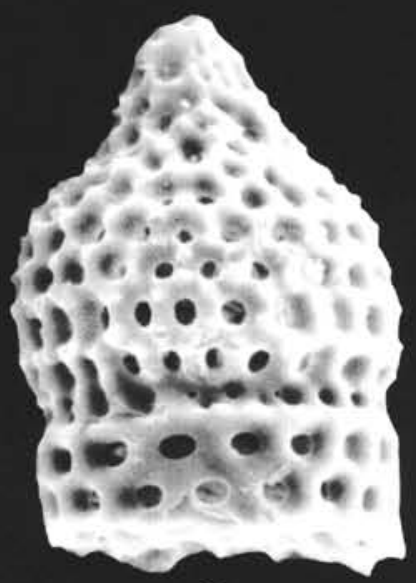

7
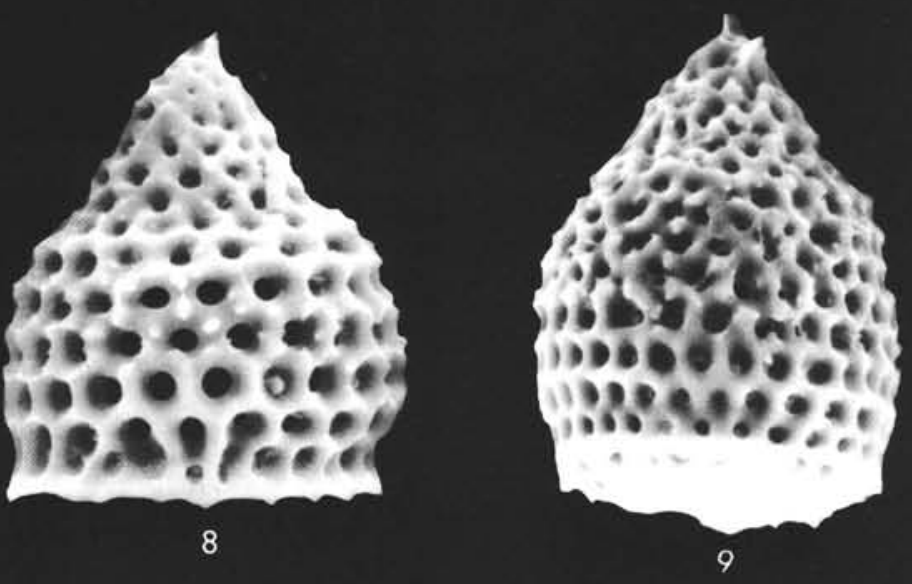


\section{PLATE 8}

Figure 1 Eucyrtidium calvertense Martin.

Sample 323-1-4, 142-144 cm; $\times 290$.

Figure 2 Lithomelissa sp. C. Chen.

ELTANIN 34-5, $400 \mathrm{~cm} ; \times 380$.

Figure 3 Lithomelissa sp. C. Chen.

ELTANIN $34-5,360 \mathrm{~cm} ; \times 480$.

Figure 4 Eucyrtidium punctatum (Ehrenberg) group. Sample 323-6, CC; $\times 350$.

Figure 5 Eucyrtidium punctatum (Ehrenberg) group. Sample 323-6, CC; $\times 330$.

Figure 6 Eucyrtidium punctatum (Ehrenberg) group. Sample 323-6, CC; $\times 320$.

Figure $7 \quad$ Eucyrtidium cienkowskii Haeckel group. Sample 323-3, CC; $\times 320$.

Figure 8 Eucyrtidium cienkowskii Haeckel group. Sample 323-6, CC; $\times 435$.

Figure 9 Eucyrtidium cienkowskii Haeckel group. Sample 323-6, CC; $\times 425$. 

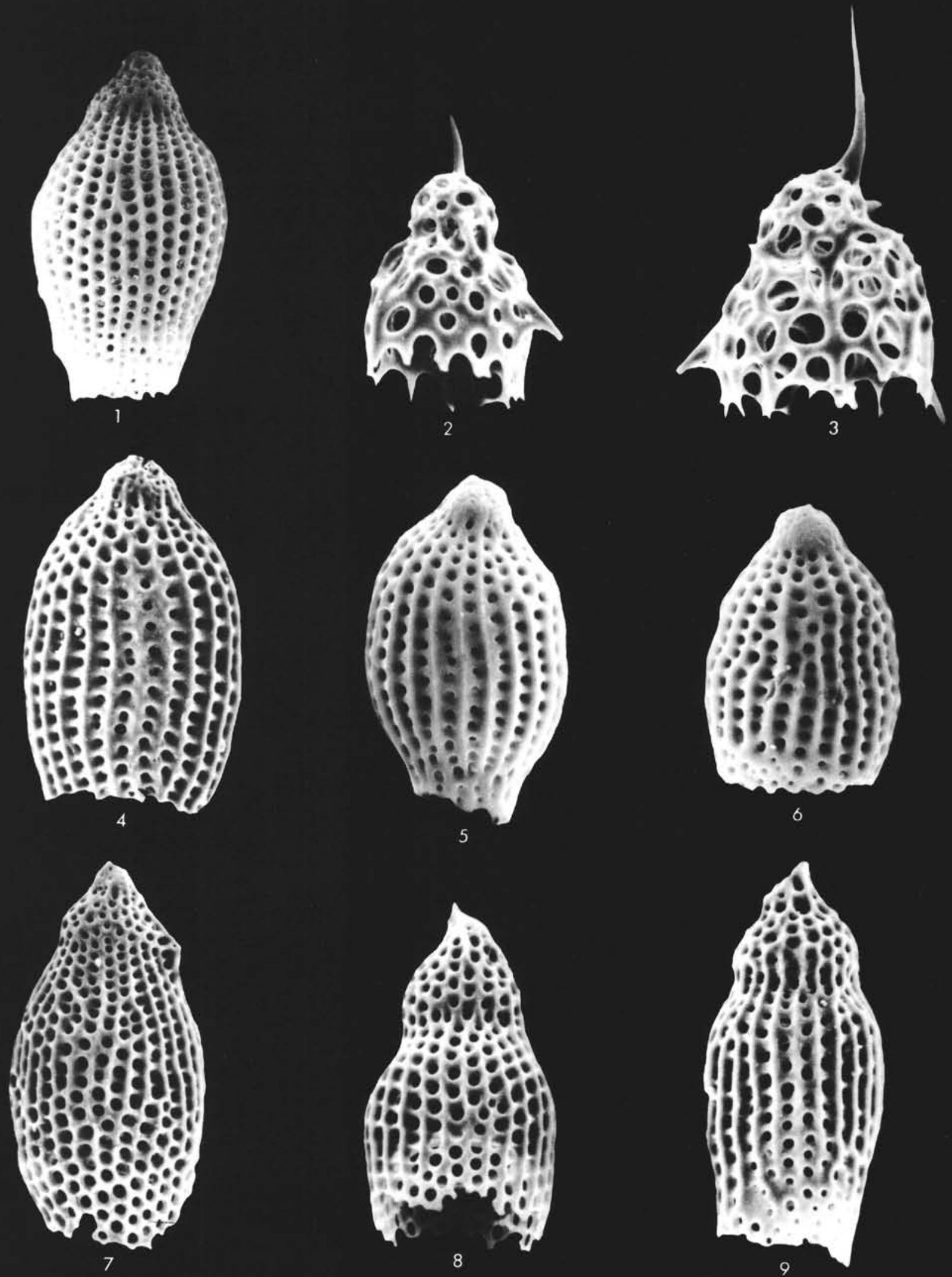


\section{PLATE 9}

Figure $1 \quad$ Cyrtocapsella tetrapera Haeckel. Sample 323-3, CC; $\times 400$.

Figure 2 Cyrtocapsella tetrapera Haeckel. Sample 323-6, CC; $\times 360$.

Figure 3 Cyrtocapsella tetrapera Haeckel. Sample 323-5, CC; $\times 380$.

Figure $4 \quad$ Cyrtocapsella isopera Chen. Sample 323-6, CC; $\times 370$.

Figure $5 \quad$ Lychnocanoma grande rugosum (Riedel). Sample 323-2, CC; $\times 225$.

Figure 6 Cyrtocapsella isopera Chen. Sample 323-3, CC; $\times 600$.

Figure 7 Cyrtocapsella tetrapera Haeckel. Sample 323-3, CC; $\times 390$.

Figure $8 \quad$ Lophocyrtis golli Chen. Sample 323-6, CC; $\times 365$.

Figure $9 \quad$ Lophocyrtis golli Chen. Sample 323-6, CC; $\times 315$. 


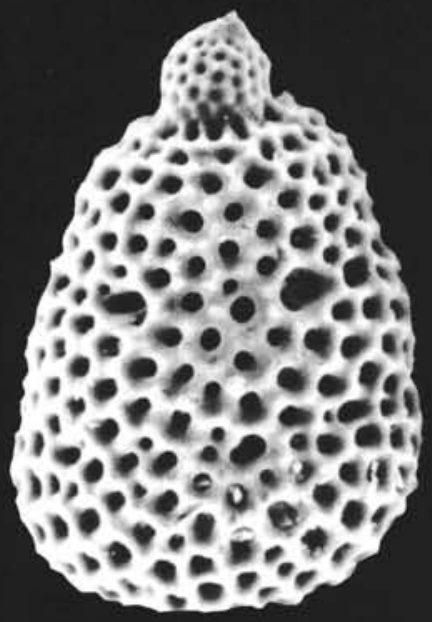

1

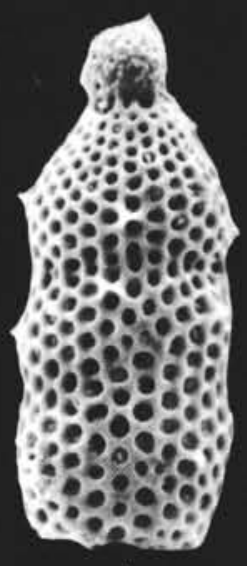

4

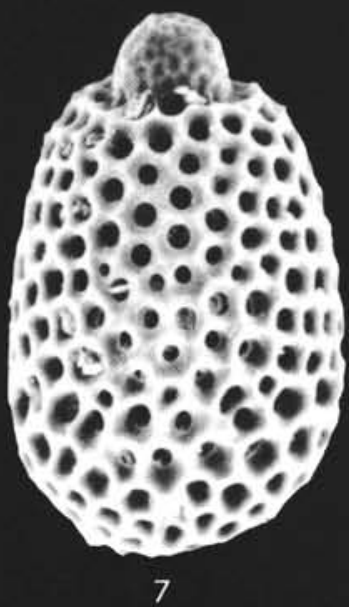

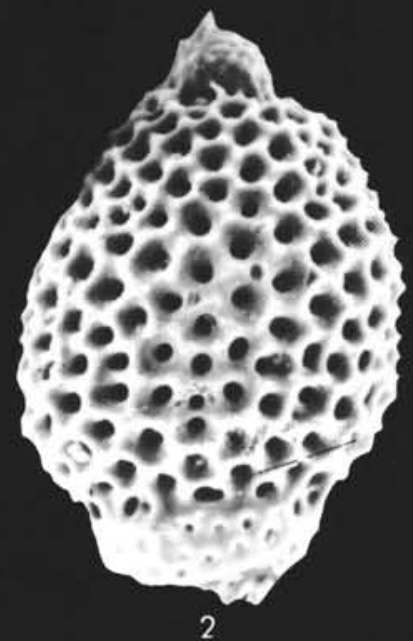

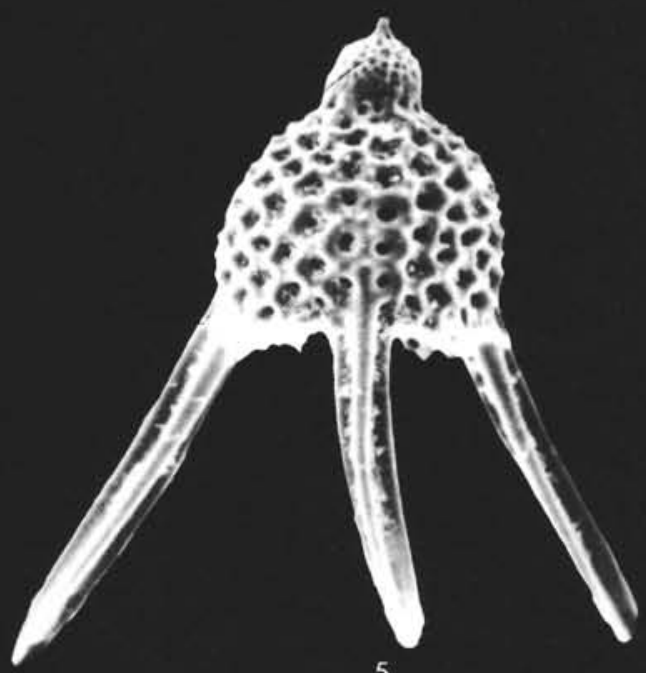

5
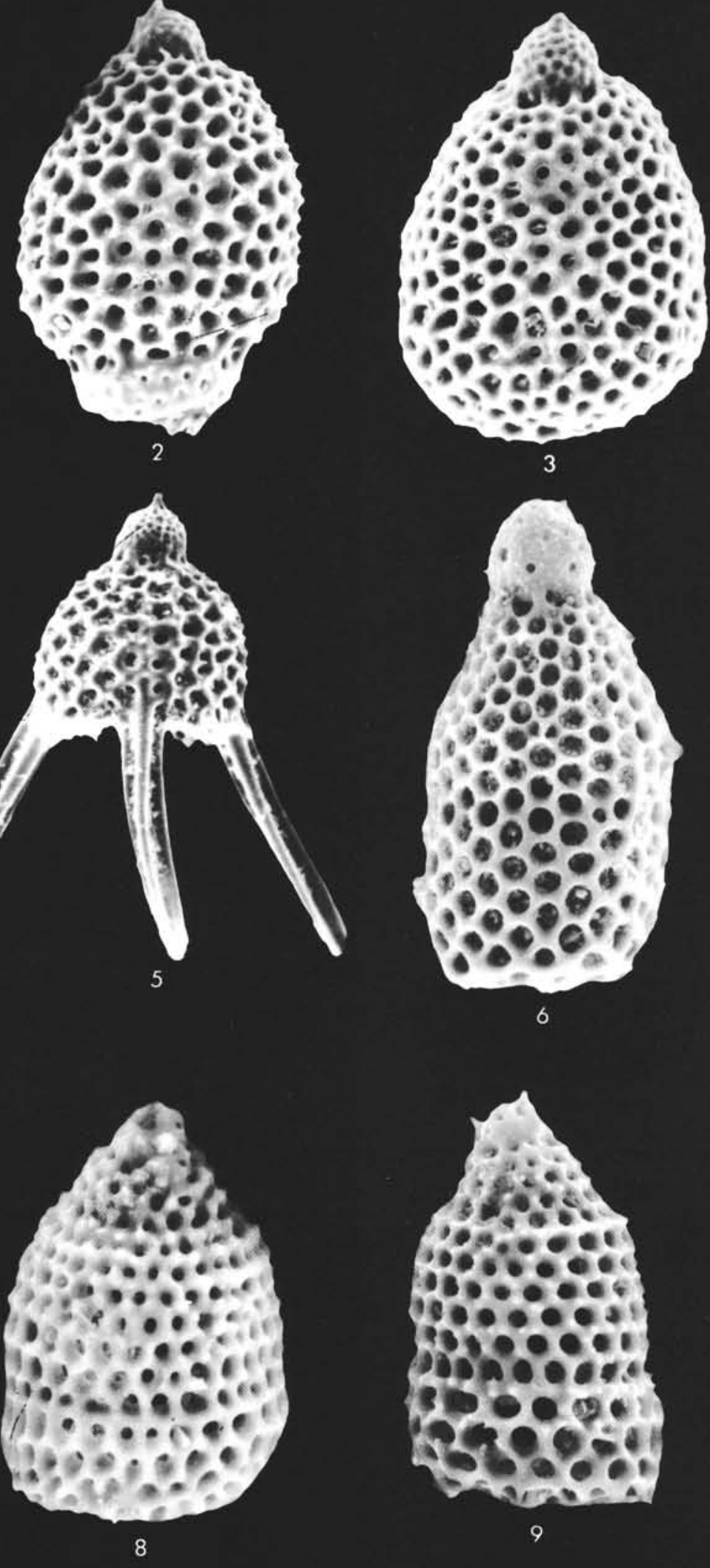